Proceedings of the Edinburgh Mathematical Society (2004) 47, 69-100 (C)

DOI:10.1017/S0013091503000038 Printed in the United Kingdom

\title{
THE CONTACT NUMBER OF A EUCLIDEAN SUBMANIFOLD
}

\author{
BANG-YEN CHEN ${ }^{1}$ AND SHI-JIE LI ${ }^{2}$ \\ ${ }^{1}$ Department of Mathematics, Michigan State University, \\ East Lansing, MI 48824-1027, USA (bychen@math.msu.edu) \\ ${ }^{2}$ Department of Mathematics, South China Normal University, \\ Guangzhou 510631, People's Republic of China (lisj@scnu.edu.cn)
}

(Received 21 January 2003)

\begin{abstract}
We introduce an invariant, called the contact number, associated with each Euclidean submanifold. We show that this invariant is, surprisingly, closely related to the notions of isotropic submanifolds and holomorphic curves. We are able to establish a simple criterion for a submanifold to have any given contact number. Moreover, we completely classify codimension-2 submanifolds with contact number $\geqslant 3$. We also study surfaces in $\mathbb{E}^{6}$ with contact number $\geqslant 4$. As an immediate consequence, we obtain the first explicit examples of non-spherical pseudo-umbilical surfaces in Euclidean spaces.
\end{abstract}

Keywords: contact number; Euclidean submanifold; isotropy; constant isotropy; normal sections; geodesic

2000 Mathematics subject classification: Primary 53C40; 53A10

Secondary 53B25; 53C42

\section{The contact number}

Throughout this paper, manifolds are assumed to be connected and without boundary and each Euclidean submanifold is of dimension $\geqslant 2$. For a Riemannian manifold $M$, we denote by $U M$ the unit tangent bundle of $M$.

Let $M$ be an $n$-dimensional submanifold in $\mathbb{E}^{m}$. For a given point $p \in M$ and a given $u \in U_{p} M$, there is a unique unit speed geodesic $\gamma_{u}$ in $M$ through $p$ satisfying $\gamma_{u}(0)=p$ and $\gamma_{u}^{\prime}(0)=u$. For the same pair $(p, u)$, there is another canonical unit speed curve $\beta_{u}$ associated with $(p, u)$ which is called the normal section (see [7]) defined as follows. Let $E(p, u)$ be the affine $(m-n+1)$-subspace of $\mathbb{E}^{m}$ through $p$ spanned by $u$ and the normal space $T_{p}^{\perp} M$ at $p$. The intersection of $M$ and $E(p, u)$ gives rise to a unit speed curve $\beta_{u}(s)$ with $\beta_{u}(0)=p$ and $\beta_{u}^{\prime}(0)=u$ defined on an open interval containing 0 . This curve $\beta_{u}$ is called the normal section at $(p, u)$.

Normal sections have been studied by many geometers (see, for example, $[\mathbf{1}, \mathbf{2}, \mathbf{7}, \mathbf{1 0}$ $\mathbf{1 5}, \mathbf{1 7}-\mathbf{2 1}, \mathbf{2 5}, \mathbf{2 6}])$. In particular, Sánchez et al. showed that normal sections play some important roles in algebraic geometry as well as in differential geometry. 
The geodesic $\gamma_{u}$ and the normal section $\beta_{u}$ at $(p, u)$ are said to be in contact of order $k$ if $\gamma_{u}^{(i)}(0)=\beta_{u}^{(i)}(0)$ for $i=1, \ldots, k$, where $\gamma_{u}^{(i)}$ and $\beta_{u}^{(i)}$ denote the $i$ th derivatives of $\gamma_{u}$ and $\beta_{u}$ with respect to their arclength functions.

In this paper we introduce the notion of contact number as follows.

Definition 1.1. A submanifold $M$ in a Euclidean space is said to be in contact of order $k$ if, for each $p \in M$ and $u \in U_{p} M$, the geodesic $\gamma_{u}$ and the normal section $\beta_{u}$ at $(p, u)$ are in contact of order $k$. If the submanifold $M$ is in contact of order $k$ for every natural number $k$, the contact number $\mathfrak{c}_{\#}(M)$ of $M$ is defined to be $\infty$. Otherwise, the contact number $\mathfrak{c}_{\#}(M)$ is defined to be the largest natural number $k$ such that $M$ is in contact of order $k$ and but not of order $k+1$.

In this paper we show that the contact number is, surprisingly, closely related to the notions of isotropic submanifolds and holomorphic curves. We prove that the contact number of each submanifold is at least 2 ; and it is at least 3 (respectively, 4) if and only if the submanifold is isotropic (respectively, constant isotropic). We also prove that a surface in a Euclidean space has contact number 3 if and only if it is a nonplanar holomorphic curve in a complex 2-plane $\mathbb{C}^{2}$. Also, we establish a simple criterion for a submanifold to have any given contact number. Moreover, we completely classify codimension- 2 submanifolds with contact number $\geqslant 3$. We also investigate surfaces in $\mathbb{E}^{6}$ with contact number $\geqslant 4$. As a consequence, we obtain the first explicit examples of non-spherical pseudo-umbilical surfaces in Euclidean spaces.

\section{Basic notation and formulae}

Let $M$ be an $n$-dimensional submanifold in $\mathbb{E}^{m}$. We choose a local field of orthonormal frames $\left\{e_{1}, \ldots, e_{m}\right\}$ in $\mathbb{E}^{m}$ such that, restricted to $M, e_{1}, \ldots, e_{n}$ are tangent to $M$ and $e_{n+1}, \ldots, e_{m}$ are normal to $M$. We denote by $\nabla$ and $\tilde{\nabla}$ the Levi-Civita connections on $M$ and $\mathbb{E}^{m}$, respectively. Let $D$ denote the normal connection of $M$ in $\mathbb{E}^{m}$.

In the following, we use the following convention on the range of indices unless mentioned otherwise:

$$
1 \leqslant i, j, k, \ell \leqslant n ; \quad n+1 \leqslant r, s, t \leqslant m ; \quad 1 \leqslant A, B, C \leqslant m .
$$

We denote by $\omega^{1}, \ldots, \omega^{m}$ the field of dual frames. The structure equations of $\mathbb{E}^{m}$ are given by

$$
\begin{gathered}
\tilde{\nabla} e_{A}=\sum \omega_{A}^{B} e_{B}, \quad \omega_{A}^{B}+\omega_{B}^{A}=0, \\
\mathrm{~d} \omega^{A}=-\sum \omega_{B}^{A} \wedge \omega^{B}, \quad \mathrm{~d} \omega_{B}^{A}=-\sum \omega_{C}^{A} \wedge \omega_{B}^{C} .
\end{gathered}
$$

Restricting these forms on $M$, we have $\omega^{r}=0,0=\mathrm{d} \omega^{r}=-\sum \omega_{i}^{r} \wedge \omega^{i}$. Thus, by applying Cartan's Lemma, we may write

$$
\omega_{i}^{r}=\sum h_{i j}^{r} \omega^{j}, \quad h_{i j}^{r}=h_{j i}^{r} .
$$

The second fundamental form $h$ of $M$ in $\mathbb{E}^{m}$ is given by $h=\sum h_{i j}^{r} \omega^{i} \omega^{j} e_{r}$. 
For any two vectors $x, y$ tangent to $M$ and any vector $\xi$ normal to $M$ we have

$$
\begin{aligned}
& \tilde{\nabla}_{x} y=\nabla_{x} y+h(x, y), \\
& \tilde{\nabla}_{x} \xi=-A_{\xi} x+D_{x} \xi,
\end{aligned}
$$

where $A_{\xi}$ is the shape operator of $M$ in $\mathbb{E}^{m}$ with respect to $\xi$. The second fundamental form and the shape operator are related by $\left\langle A_{\xi} x, y\right\rangle=\langle h(x, y), \xi\rangle$.

The covariant derivative $\bar{\nabla} h$ of $h$ with respect to $T M \oplus T^{\perp} M$ is defined by

$$
\left(\bar{\nabla}_{x} h\right)(y, z)=D_{x} h(y, z)-h\left(\nabla_{x} y, z\right)-h\left(y, \nabla_{x} z\right) .
$$

Sometimes, we write $\left(\bar{\nabla}_{x} h\right)(y, z)$ as $(\bar{\nabla} h)(y, z, x)$. We put $\bar{\nabla}^{0} h=h$.

In general, the $k$ th $(k \geqslant 1)$ covariant derivative $\bar{\nabla}^{k} h$ of $h$ is given by

$$
\begin{aligned}
\left(\bar{\nabla}^{k} h\right)\left(x_{1}, x_{2}, \ldots, x_{k+2}\right)=D_{x_{k+2}}\left(\left(\bar{\nabla}^{k-1} h\right)\left(x_{1}, \ldots, x_{k+1}\right)\right) & \\
& \quad-\sum_{i=1}^{k+1}\left(\bar{\nabla}^{k-1} h\right)\left(x_{1}, \ldots, \nabla_{x_{k+2}} x_{i}, \ldots, x_{k+1}\right) .
\end{aligned}
$$

It is clear that $\bar{\nabla}^{k} h$ is a normal-bundle-valued tensor field of type $(0, k+2)$. We simply denote

$$
\left(\bar{\nabla}^{q} h\right)(\overbrace{x, \ldots, x}^{q+2})
$$

by $\left(\bar{\nabla}^{q} h\right)\left(x^{q+2}\right)$. From (2.6) and (2.7), we have

$$
\begin{aligned}
& D_{x} h(x, x)=(\bar{\nabla} h)\left(x^{3}\right)+2 h\left(x, \nabla_{x} x\right), \\
& D_{x}^{2} h(x, x)=\left(\bar{\nabla}^{2} h\right)\left(x^{4}\right)+5\left(\bar{\nabla}_{x} h\right)\left(x, \nabla_{x} x\right)+2 h\left(\nabla_{x} x, \nabla_{x} x\right)+2 h\left(x, \nabla_{x}^{2} x\right) .
\end{aligned}
$$

The equations of Gauss, Codazzi and Ricci are given, respectively, by

$$
\begin{aligned}
R(x, y, z, w) & =\langle h(x, w), h(y, z)\rangle-\langle h(x, z), h(y, w)\rangle, \\
\left(\bar{\nabla}_{x} h\right)(y, z) & =\left(\bar{\nabla}_{y} h\right)(x, z), \\
R^{D}(x, y, \xi, \eta) & =\left\langle\left[A_{\xi}, A_{\eta}\right](x), y\right\rangle
\end{aligned}
$$

where $R(x, y)=\nabla_{x} \nabla_{y}-\nabla_{y} \nabla_{x}-\nabla_{[x, y]}$ and $R^{D}(x, y)=D_{x} D_{y}-D_{y} D_{x}-D_{[x, y]}$ are the curvature tensors of the tangent and normal bundles.

\section{Lemmas and examples}

We recall the following definition from $[\mathbf{2 2}]$.

Definition 3.1. A submanifold $M$ in a Riemannian manifold is said to be isotropic if, for each point $p \in M$, the length $\lambda=|h(u, u)|$ of the normal curvature vector $h(u, u)$ is independent of the choice of $u \in U_{p} M$. If $\lambda=|h(u, u)|$ is also independent of $p \in M$, then $M$ is said to be constant isotropic. 
We need the following result for later use.

Lemma 3.2 (see [22]). A submanifold $M$ is isotropic if and only if we have

$$
\langle h(u, u), h(u, v)\rangle=0
$$

for orthonormal vectors $u, v$ tangent to $M$ at each point.

For isotropic submanifolds, we also have

$$
\begin{aligned}
\langle h(u, u), h(v, v)\rangle+2\langle h(u, v), h(u, v)\rangle & =|h(u, u)|^{2}, \\
\langle h(u, u), h(v, w)\rangle+2\langle h(u, v), h(u, w)\rangle & =0
\end{aligned}
$$

for orthonormal vectors $u, v, w$ tangent to $M$ at each point.

We also need the following lemma for constant isotropic submanifolds.

Lemma 3.3. An isotropic submanifold $M$ is constant isotropic if and only if we have

$$
\left\langle A_{(\bar{\nabla} h)\left(u^{3}\right)} u, v\right\rangle=0
$$

for orthonormal vectors $u, v$ tangent to $M$ at each point.

Proof. Assume that $M$ is an isotropic submanifold, so we have $|h(u, u)|^{2}=\lambda^{2}(p)$ for each unit vector $u \in T_{p} M$. For any orthonormal vectors $u, v$ in $T_{p} M$, we extend $u$ and $v$ to orthonormal vector fields $X$ and $Y$ on some open neighbourhood of $p$ such that $\nabla_{u} X=\nabla_{u} Y=\nabla_{v} X=0$ at $p$. Since $M$ is isotropic, we obtain from (2.6) and (2.11) that

$$
\begin{aligned}
v \lambda^{2} & =v\left(|h(X, X)|^{2}\right)=2\left\langle D_{v} h(X, X), h(X, X)\right\rangle \\
& =2\left\langle\left(\bar{\nabla}_{v} h\right)(u, u), h(u, u)\right\rangle=2\left\langle\left(\bar{\nabla}_{u} h\right)(u, v), h(u, u)\right\rangle \\
& =2\left\langle D_{u} h(X, Y), h(X, X)\right\rangle=-2\left\langle D_{u} h(X, X), h(X, Y)\right\rangle \\
& =-2\left\langle\left(\bar{\nabla}_{u} h\right)(u, u), h(u, v)\right\rangle .
\end{aligned}
$$

Since $\operatorname{dim} M$ is at least two, (3.5) implies the lemma.

Similarly, we also have

$$
u \lambda^{2}=2\left\langle\left(\bar{\nabla}_{u} h\right)(u, u), h(u, u)\right\rangle,
$$

for any isotropic submanifold.

Lemma 3.4. An isotropic submanifold $M$ is constant isotropic if and only if we have

$$
A_{(\bar{\nabla} h)\left(u^{3}\right)} u=0
$$

for any vector $u$ tangent to $M$ at each point. 
Proof. If $M$ is constant isotropic, then (3.6) implies

$$
\left\langle A_{\left(\bar{\nabla}_{u} h\right)(u, u)} u, u\right\rangle=0 .
$$

Combining Lemma 3.3 and (3.8) gives (3.7). The converse follows from Lemma 3.3.

A Euclidean submanifold $M$ is said to have geodesic normal sections if every normal section on $M$ is a geodesic (see [10]). All submanifolds $M$ in $\mathbb{E}^{m}$ with geodesic normal sections satisfy $\mathfrak{c}_{\#}(M)=\infty$. A Euclidean submanifold $M$ is called helical if geodesics of $M$, considered as curves in $\mathbb{E}^{m}$, have all Frenet curvatures constant and independent of the chosen geodesic. Helical immersions have been studied extensively (see, for example, $[18,24])$.

Chen and Verheyen proved the following results for submanifolds with geodesic normal sections.

Theorem A (see [10]). Every submanifold in $\mathbb{E}^{m}$ with geodesic normal sections is constant isotropic.

Theorem B (see $[\mathbf{1 0}, \mathbf{2 6}])$. A submanifold in $\mathbb{E}^{m}$ has geodesic normal sections if and only if it is helical.

Example 3.5. Let $M$ be a compact Riemannian manifold. Then $M$ has a unique kernel of the heat equation: $K: M \times M \times \mathbb{R}_{0}^{+} \rightarrow \mathbb{R}$. Let $\delta$ denote the distance function on $M$. Then $M$ is called a strongly harmonic manifold if there exists a function $\Psi: \mathbb{R}^{+} \times \mathbb{R}_{0}^{+} \rightarrow \mathbb{R}$ such that $K(x, y, t)=\Psi(\delta(x, y), t)$ for $x, y \in M$ and $t \in \mathbb{R}_{0}^{+}$. Compact symmetric spaces of rank one are known examples of strongly harmonic manifolds [3, p. 158].

Let $\lambda_{k}$ be the $k$ th non-zero eigenvalue of the Laplacian $\Delta$. Denote by $V_{k}$ be the eigenspace of $\Delta$ with eigenvalue $\lambda_{k}$. On $V_{k}$ we define an inner product by $\langle\langle f, g\rangle\rangle=$ $\int_{M} f g * 1$ for $f, g \in V_{k} . V_{k}$ together with $\langle\langle\cdot, \cdot\rangle\rangle$ is a finite-dimensional Euclidean space. Let $\varphi_{k}^{1}, \ldots, \varphi_{k}^{m}$ be an orthonormal basis of $V_{k}$. Then the mapping

$$
\varphi_{k}: M \rightarrow \mathbb{E}^{m}: x \mapsto c_{k}\left(\varphi_{k}^{1}(x), \ldots, \varphi_{k}^{m}(x)\right)
$$

defines a helical isometric immersion for some suitable constant $c_{k}$. Such submanifolds satisfy $\mathfrak{c}_{\#}(M)=\infty$.

In particular, if $M=S^{2}(\sqrt{3} / a)$ denotes the 2-sphere with constant sectional curvature $3 / a^{2}$, then $\varphi_{2}^{a}: S^{2}(\sqrt{3} / a) \rightarrow S^{4}(1 / a) \subset \mathbb{E}^{5}$ is given by

$$
\varphi_{2}^{a}=a\left(\frac{1}{\sqrt{3}} y z, \frac{1}{\sqrt{3}} x z, \frac{1}{\sqrt{3}} x y, \frac{1}{2 \sqrt{3}}\left(x^{2}-y^{2}\right), \frac{1}{6}\left(x^{2}+y^{2}-2 z^{2}\right)\right),
$$

where $x^{2}+y^{2}+z^{2}=3$. This isometric minimal immersion of $S^{2}(\sqrt{3} / a)$ into $S^{4}(1 / a)$ is called a Veronese surface.

Example 3.6. Let $\psi_{j}: M \rightarrow \mathbb{E}^{m_{j}}(j=1, \ldots, s)$ be $s$ isometric immersions with geodesic normal sections. For any real numbers $c_{1}, \ldots, c_{s}$ with $c_{1}^{2}+\cdots+c_{s}^{2}=1$, the diagonal immersion,

$$
\left(c_{1} \psi_{1}, \ldots, c_{\ell} \psi_{s}\right): M \rightarrow \mathbb{E}^{m_{1}+\cdots+m_{s}}: p \mapsto\left(c_{1} \psi_{1}(p), \ldots, c_{s} \psi_{s}(p)\right),
$$

satisfies $\mathfrak{c}_{\#}(M)=\infty$. 


\section{Relations between contact number and isotropy}

Theorem 4.1. For every submanifold $M$ in a Euclidean space, we have

(1) the contact number $\mathfrak{c}_{\#}(M)$ of $M$ is at least 2 , i.e. $\mathfrak{c}_{\#}(M) \geqslant 2$;

(2) $M$ is isotropic if and only if $\mathfrak{c}_{\#}(M) \geqslant 3$ holds;

(3) $M$ is constant isotropic if and only if $\mathfrak{c}_{\#}(M) \geqslant 4$ holds.

Proof. Let $M$ be a submanifold of dimension $n \geqslant 2$ in $\mathbb{E}^{m}$. Then, for any unit speed curve $\alpha=\alpha(s)$ in $M$, we have

$$
\begin{aligned}
& \alpha^{\prime}(s)=T, \\
& \alpha^{\prime \prime}(s)=\nabla_{T} T+h(T, T), \\
& \alpha^{\prime \prime \prime}(s)=\nabla_{T}^{2} T+h\left(T, \nabla_{T} T\right)-A_{h(T, T)} T+D_{T} h(T, T), \\
& \alpha^{\mathrm{iv}}(s)=\nabla_{T}^{3} T-3 A_{h\left(T, \nabla_{T} T\right)} T-\nabla_{T}\left(A_{h(T, T)} T\right)-A_{\left(\bar{\nabla}_{T} h\right)(T, T)} T \\
& +h\left(T, \nabla_{T}^{2} T\right)+D_{T} h\left(T, \nabla_{T} T\right)-h\left(T, A_{h(T, T)} T\right)+D_{T}^{2} h(T, T),
\end{aligned}
$$

where $T=\alpha^{\prime}(s)$ is the unit vector field tangent to $\alpha$ and $\nabla_{T}^{2} T=\nabla_{T} \nabla_{T} T, \ldots$, etc.

Using (2.6), (2.8) and (2.9), Equation (4.4) can be rewritten as

$$
\begin{aligned}
\alpha^{\mathrm{iv}}(s)= & \nabla_{T}^{3} T-3 A_{h\left(T, \nabla_{T} T\right)} T-\nabla_{T}\left(A_{h(T, T)} T\right)-A_{\left(\bar{\nabla}_{T} h\right)(T, T)} T+\left(\bar{\nabla}^{2} h\right)\left(T^{4}\right) \\
& +6(\bar{\nabla} h)\left(T, T, \nabla_{T} T\right)-h\left(T, A_{h(T, T)} T\right)+4 h\left(T, \nabla_{T}^{2} T\right)+3 h\left(\nabla_{T} T, \nabla_{T} T\right) .
\end{aligned}
$$

Let $\gamma_{u}(s)$ and $\beta_{u}(s)$ denote, respectively, the unique geodesic and the unique normal section associated with a point $p \in M$ and a vector $u \in U_{p} M$ so that $\gamma_{u}(0)=\beta_{u}(0)=p$ and $\gamma_{u}^{\prime}(0)=\beta_{u}^{\prime}(0)=u$. For the geodesic $\gamma_{u}$, we have $\nabla_{T} T=0$ along $\gamma_{u}$, with $T_{\gamma}(s)=$ $\gamma_{u}^{\prime}(s)$. Thus we obtain from (4.2) and (4.3) that

$$
\begin{aligned}
\gamma_{u}^{\prime \prime}(0) & =h(u, u), \\
\gamma_{u}^{\prime \prime \prime}(0) & =-A_{h(u, u)} u+\left(\bar{\nabla}_{u} h\right)(u, u) .
\end{aligned}
$$

On the other hand, for the normal section $\beta_{u}$ with $\beta_{u}(0)=p$ and $\beta_{u}^{\prime}(0)=u$, we obtain from (4.2) that

$$
\begin{gathered}
\nabla_{u} T_{\beta}=0, \quad T_{\beta}=\beta_{u}^{\prime}(s), \\
\beta_{u}^{\prime \prime}(0)=h(u, u),
\end{gathered}
$$

since $\beta_{u}^{\prime \prime}(0)$ lies in $E(p, u)$, which is spanned by $u$ and the normal space $T_{p}^{\perp} M$.

Comparing (4.6) and (4.9), we find $\gamma_{u}^{\prime \prime}(0)=\beta_{u}^{\prime \prime}(0)$. Hence, $M$ is in contact of order at least 2. This proves statement (1).

Now, suppose that the contact number of $M$ is at least 3 . Then we have $\gamma_{u}^{\prime \prime \prime}(0)=\beta_{u}^{\prime \prime \prime}(0)$. Since $\beta_{u}^{\prime \prime \prime}(0)$ lies in $E(p, u)$, we obtain from (4.7) that $A_{h(u, u)} u$ lies in $E(p, u)$. Hence, we obtain $\langle h(u, u), h(u, v)\rangle=0$ for any orthonormal vectors $u, v \in T_{p} M$. Because this is true 
for any point $p$ and any orthonormal vectors $u, v$ at $p$, Lemma 3.2 implies that $M$ is isotropic.

Conversely, if $M$ is isotropic, Lemma 3.2 implies that

$$
A_{h(u, u)} u=\lambda_{0} u
$$

for some number $\lambda_{0}$. Hence, we obtain from (4.7) that

$$
\gamma_{u}^{\prime \prime \prime}(0)=-\lambda_{0} u+\left(\bar{\nabla}_{u} h\right)(u, u) .
$$

For the normal section $\beta_{u}$, we obtain from (4.3), (4.8) and (4.10) that

$$
\beta_{u}^{\prime \prime \prime}(0)=\nabla_{u} \nabla_{T_{\beta}} T_{\beta}-\lambda_{0} u+\left(\bar{\nabla}_{u} h\right)(u, u) .
$$

Since $\beta_{u}^{\prime \prime \prime}(o)$ lies in $E(p, u),(4.12)$ implies

$$
\left\langle\nabla_{u} \nabla_{T_{\beta}} T_{\beta}, v\right\rangle=0
$$

for orthonormal $u, v \in T_{p} M$. On the other hand, we find from (4.8) that

$$
2\left\langle\nabla_{u} \nabla_{T_{\beta}} T_{\beta}, u\right\rangle=u\left(T_{\beta}\left\langle T_{\beta}, T_{\beta}\right\rangle\right)-2\left\langle\nabla_{u} T_{\beta}, \nabla_{u} T_{\beta}\right\rangle=0 .
$$

Combining (4.13) and (4.14) yields

$$
\nabla_{u} \nabla_{T_{\beta}} T_{\beta}=0 .
$$

Using (4.11), (4.12) and (4.15), we obtain $\gamma_{u}^{\prime \prime \prime}(0)=\beta_{u}^{\prime \prime \prime}(0)$, which implies that the contact number is at least 3 . This proves statement (2).

Now, let us assume that $M$ is an isotropic submanifold. Then we have

$$
A_{h(u, u)} u=\lambda_{0} u
$$

where $\lambda_{0}$ is independent of the choice of $u \in U_{p} M$ for each $p \in M$.

From (4.5), (4.16) and $\nabla_{T_{\gamma}} T_{\gamma}=0$, we know that the geodesic $\gamma_{u}$ satisfies

$$
\gamma_{u}^{\mathrm{iv}}(0)=-\left(u \lambda_{0}\right) u-A_{\left(\bar{\nabla}_{u} h\right)(u, u)} u+\left(\bar{\nabla}^{2} h\right)\left(u^{4}\right)-\lambda_{0} h(u, u) .
$$

If $\mathfrak{c}_{\#}(M)$ is at least 4 , we have $\gamma_{u}^{\text {iv }}(0)=\beta_{u}^{\text {iv }}(0)$. Since $\beta_{u}^{\text {iv }}(0)$ lies in $E(p, u),(4.17)$ implies that $\left\langle A_{(\bar{\nabla} h)\left(u^{3}\right)} u, v\right\rangle=0$ for orthonormal vectors $u, v \in T_{p} M$. Hence, Lemma 3.3 implies that $M$ is constant isotropic.

Conversely, assume that $M$ is constant isotropic. Then, by Lemma 3.3, we have

$$
A_{(\bar{\nabla} h)\left(u^{3}\right)} u=\lambda_{1} u
$$

for any unit vector $u$ tangent to $M$ at each point, where $\lambda_{1}$ is a function on $M$.

For $\beta_{u}$, we obtain from (4.5), (4.8), (4.10), (4.15) and (4.18) that

$$
\beta_{u}^{\mathrm{iv}}(0)=\nabla_{u} \nabla_{T_{\beta}}^{2} T_{\beta}-\left(u \lambda_{0}\right) u-\lambda_{1} u+\left(\bar{\nabla}^{2} h\right)\left(u^{4}\right)-\lambda_{0} h(u, u) .
$$

Since $\beta_{u}^{\text {iv }}(0)$ lies in $E(p, u),(4.19)$ implies that $\nabla_{u} \nabla_{T_{\beta}}^{2} T_{\beta}$ is parallel to $u$. 
On the other hand, since $s=0$ is a critical point of $\left|\nabla_{T_{\beta}} T_{\beta}\right|^{2}$ by (4.8), we find

$$
u\left\langle\nabla_{T_{\beta}}^{2} T_{\beta}, T_{\beta}\right\rangle=u\left\{T_{\beta}\left\langle\nabla_{T_{\beta}} T_{\beta}, T_{\beta}\right\rangle-\left|\nabla_{T_{\beta}} T_{\beta}\right|^{2}\right\}=0 .
$$

Using (4.8) and (4.20) we get

$$
\left\langle\nabla_{u} \nabla_{T_{\beta}}^{2} T_{\beta}, u\right\rangle=u\left\langle\nabla_{T_{\beta}}^{2} T_{\beta}, T_{\beta}\right\rangle-\left\langle\nabla_{T_{\beta}}^{2} T_{\beta}, \nabla_{u} T_{\beta}\right\rangle=0 .
$$

Hence, we get $\nabla_{u} \nabla_{T_{\beta}}^{2} T_{\beta}=0$. Combining this with (4.17) and (4.19) yields $\gamma_{u}^{v}(0)=\beta_{u}^{v}(0)$. Thus, $\mathfrak{c}_{\#}(M)$ is at least 4 . This proves statement (3).

\section{Classification of codimension-2 submanifolds with $\mathfrak{c}_{\#}(M) \geqslant 3$}

Theorem 5.1. Let $M$ be an $n$-dimensional submanifold of $\mathbb{E}^{n+2}$. Then $\mathfrak{c}_{\#}(M) \geqslant 3$ holds if and only if one of the following three cases occurs.

(1) $\mathfrak{c}_{\#}(M)=3, n=2$, and $M$ is a complex curve lying linearly fully in $\mathbb{C}^{2}$, where $\mathbb{C}^{2}$ denotes $\mathbb{E}^{4}$ endowed with some orthogonal complex structure.

(2) $\mathfrak{c}_{\#}(M)=\infty$ and $M$ is an open portion of an $n$-plane.

(3) $\mathfrak{c}_{\#}(M)=\infty$ and $M$ is an open portion of a hypersphere lying in a hyperplane of $\mathbb{E}^{n+2}$.

Proof. Suppose that $\mathfrak{c}_{\#}(M) \geqslant 3$. Then $M$ is isotropic according to Theorem 4.1. Thus, we have $|h(u, u)|=\lambda(p)$ for every $u \in U_{p} M$.

Case (a). $h(u, v)=0$ for any orthonormal vectors $u, v$ tangent to $M$. In this case, $M$ is totally umbilical. So, we have either Case (2) or Case (3) (cf. [6]).

Case (b). $h(u, v) \neq 0$ for some orthonormal vectors $u, v$ tangent to $M$. We put

$$
U=\left\{p \in M: h(u, v) \neq 0 \text { for some orthonormal vectors } u, v \in T_{p} M\right\} .
$$

Clearly, $U$ is a non-empty open subset $U$ which is non-totally umbilical at every point. On $U$, let $e_{1}=u$ and $e_{n}=v$. We extend $e_{1}, e_{n}$ to a local field of orthonormal frames $e_{1}, \ldots, e_{n}$. So, by Lemma 3.2 , we may put

$$
h\left(e_{1}, e_{1}\right)=\lambda e_{n+1}, \quad h\left(e_{1}, e_{n}\right)=\mu e_{n+2}, \quad \lambda, \mu>0 .
$$

If $h\left(e_{1}, e_{j}\right)=0$ for some $j \in\{2, \ldots, n-1\}$, then (3.1), (3.2) and the isotropic condition imply that $h\left(e_{j}, e_{j}\right)=\lambda e_{n+1}$.

If $h\left(e_{1}, e_{j}\right) \neq 0$ for some $j \in\{2, \ldots, n\}$, then (3.1) implies that $h\left(e_{1}, e_{j}\right)$ is parallel to $e_{n+2}$; and hence $h\left(e_{j}, e_{j}\right)$ is parallel to $e_{n+1}$. Thus, by (3.2) and the isotropy, we get $h\left(e_{j}, e_{j}\right)=-\lambda e_{n+1}$ and $h\left(e_{1}, e_{j}\right)= \pm \lambda e_{n+2}$. Without loss of generality, we may assume that

$$
h\left(e_{1}, e_{2}\right)=\cdots=h\left(e_{1}, e_{\ell}\right)=0, \quad h\left(e_{1}, e_{j}\right)= \pm \lambda e_{n+2}, \quad j=\ell+1, \ldots, n,
$$


for some $\ell \in\{2, \ldots, n-1\}$. If $h\left(e_{1}, e_{n-1}\right) \neq 0$, then (3.1) and (3.2) imply that

$$
h\left(e_{n-1}, e_{n-1}\right)=-\lambda e_{n+1} \quad \text { and } \quad h\left(e_{n-1}, e_{n}\right)=0
$$

on $U$. Hence, by (3.3), we find $\left\langle h\left(e_{1}, e_{n-1}\right), h\left(e_{1}, e_{n}\right)\right\rangle=0$, which is a contradiction. Thus, we obtain

$$
\left.\begin{array}{ll}
h\left(e_{1}, e_{2}\right)=\cdots=h\left(e_{1}, e_{n-1}\right)=0, & h\left(e_{1}, e_{n}\right)=\lambda e_{n+2}, \\
h\left(e_{1}, e_{1}\right)=\cdots=h\left(e_{n-1}, e_{n-1}\right)=\lambda e_{n+1}, & h\left(e_{n}, e_{n}\right)=-\lambda e_{n+1} .
\end{array}\right\}
$$

Suppose that $n \geqslant 3$. Then (3.2) and (5.3) imply that $h\left(e_{2}, e_{n}\right)= \pm \lambda e_{n+2}$ on $U$. On the other hand, by (3.3) and (5.3), we get $\left\langle h\left(e_{1}, e_{n}\right), h\left(e_{2}, e_{n}\right)\right\rangle=0$, which is a contradiction. Therefore, we obtain $n=2$.

With respect to the orthonormal frame $e_{1}, e_{2}$, the shape operator satisfies

$$
A_{3}=\left(\begin{array}{cc}
\lambda & 0 \\
0 & -\lambda
\end{array}\right), \quad A_{4}=\left(\begin{array}{cc}
0 & \lambda \\
\lambda & 0
\end{array}\right)
$$

on $U$. Hence, $U$ is a minimal surface. Since each point in the complement $M-U$ of $U$ is totally umbilical, continuity and (5.4) imply that each point in $M-U$ is a totally geodesic point. Hence, the whose surface $M$ is a minimal surface.

When $\mathfrak{c}_{\#}(M) \geqslant 4, M$ is constant isotropic. So, the equation of Gauss and (5.4) imply that $M$ has constant Gauss curvature. Thus, by applying a result of $[\mathbf{5 , 2 3}], M$ is totally geodesic, which is a contradiction. Hence, we get $\mathfrak{c}_{\#}(M)=3$. Moreover, since $M$ is minimal and non-totally geodesic, $\lambda$ vanishes only at isolated points.

Let $V$ be an oriented 2-plane through the origin in $\mathbb{E}^{4}$ and $u, v$ an oriented orthonormal basis of $V$. Then $u \wedge v$ is a decomposable 2-vector of norm one which gives an orientation on $V$. Conversely, every decomposable 2-vector of norm one determines a unique 2-plane in $\mathbb{E}^{4}$ through the origin. So, if we denote by $G(2,4)$ the Grassmannian consisting of all oriented 2-planes through the origin of $\mathbb{E}^{4}$, then $G(2,4)$ can be identified naturally with the decomposable 2-vectors of norm one in the Euclidean 6 -space $\Lambda^{2} E^{4}$. Notice that the inner product on $\Lambda^{2} E^{4}$ is given by

$$
\langle u \wedge v, w \wedge z\rangle=\langle u, w\rangle\langle v, z\rangle-\langle u, z\rangle\langle v, w\rangle .
$$

Let $\phi: M \rightarrow \mathbb{E}^{4}$ be an isometric immersion of $M$ in $\mathbb{E}^{4}$. Consider the Gauss map: $\nu: M \rightarrow G(2,4) ; p \mapsto e_{1}(p) \wedge e_{2}(p)$ of $\phi$ which carries each point $p \in M$ to the 2-plane obtained from $T_{p} M$ via parallel translation. Since $\phi$ is minimal and non-totally geodesic, $\nu$ is a regular map except at some isolated points on which $\lambda$ vanishes. It follows from (5.4) that the induced metric $G$ on the Gauss image $\nu(M)$ is given by

$$
G=2 \lambda^{2} g,
$$

where $g$ is the original metric on $M$. After a direct computation, we see that the second fundamental form $h^{\nu}$ of $\nu:(M, G) \rightarrow \Lambda^{2} E^{4}$ satisfies

$$
\left.\begin{array}{l}
h^{\nu}\left(\nu_{*}\left(e_{1}\right), \nu_{*}\left(e_{1}\right)\right)=2 \lambda^{2}\left(e_{3} \wedge e_{4}-e_{1} \wedge e_{2}\right), \\
h^{\nu}\left(\nu_{*}\left(e_{1}\right), \nu_{*}\left(e_{2}\right)\right)=0 \\
h^{\nu}\left(\nu_{*}\left(e_{2}\right), \nu_{*}\left(e_{2}\right)\right)=2 \lambda^{2}\left(e_{3} \wedge e_{4}-e_{1} \wedge e_{2}\right) .
\end{array}\right\}
$$


It follows from (5.5), (5.6) and the equation of Gauss that the Gauss curvature $\tilde{K}$ of the Gauss image $(\nu(M), G)$ is 2 at every point. Thus, it follows from Theorem 6.3 of [16] that $M$ is a complex curve lying fully in $\mathbb{C}^{2}$, where $\mathbb{C}^{2}$ denotes $\mathbb{E}^{4}$ endowed with some orthogonal complex structure. The converse is easy to verify.

Corollary 5.2. Let $M$ be a hypersurface of Euclidean $(n+1)$-space $\mathbb{E}^{n+1}$. Then one of the following three cases must occur.

(1) $\mathfrak{c}_{\#}(M)=2$.

(2) $\mathfrak{c}_{\#}(M)=\infty$ and $M$ is an open portion of a hyperplane.

(3) $\mathfrak{c}_{\#}(M)=\infty$ and $M$ is an open portion of a hypersphere.

Proof. This follows immediately from Theorem 5.1.

The following result is a very simple characterization of complex curves in $\mathbb{C}^{2}$.

Corollary 5.3. A surface $M$ in $\mathbb{E}^{4}$ satisfies $\mathfrak{c}_{\#}(M)=3$ if and only if it is a non-planar holomorphic curve with respect to some orthogonal complex structure on $\mathbb{E}^{4}$.

Proof. This follows trivially from Theorem 5.1.

A surface $M$ in $\mathbb{C}^{2}$ is called Lagrangian if the complex structure $J$ of $\mathbb{C}^{2}$ interchanges each tangent space of $M$ with its corresponding normal space.

Corollary 5.4. Every non-planar minimal Lagrangian surface $M$ in the complex Euclidean plane $\mathbb{C}^{2}$ satisfies $\mathfrak{c}_{\#}(M)=3$.

Proof. This follows from Theorem 5.1 and a result of [9] which states that every minimal Lagrangian surface in $\mathbb{C}^{2}=\left(\mathbb{E}^{4}, J\right)$ is a complex curve with respect to some orthogonal complex structure on $\mathbb{E}^{4}$.

\section{A simple criterion for submanifolds to satisfy $\mathfrak{c}_{\#}(M)=k$}

Theorem 6.1. A submanifold in a Euclidean space is in contact of order $k(k \geqslant 3)$ if and only if each $u \in U M$ is an eigenvector of $A_{\left(\bar{\nabla}^{j} h\right)\left(u^{j+2}\right)}$ for $j=0, \ldots, k-3$.

Proof. When $k=3$, this follows from Lemma 3.2 and Theorem 4.1. When $k=4$, this follows from Lemma 3.3, Lemma 3.4 and Theorem 4.1. So, we only need to prove this theorem for $k \geqslant 5$. In order to do so, let us prove the following.

Lemma 6.2. If $M$ is in contact of order $k \geqslant 5$, then, for each $u \in U M$, we have

$$
A_{\left(\bar{\nabla}^{j} h\right)\left(u^{j+2}\right)} u=\lambda_{j} u, \quad j=0, \ldots, k-3,
$$

for some functions $\lambda_{j}$ on $U M$. 
Proof of Lemma 6.2. Let us assume that $M$ is in contact of order $k, k \geqslant 5$. Then Lemmas 3.2 and 3.4 and Theorem 6.1 imply that

$$
A_{h(u, u)} u=\lambda_{0} u, A_{(\bar{\nabla} h)\left(u^{3}\right)} u=0
$$

for each $u \in U M$, where $\lambda_{0}$ is constant. It follows from (4.5) and (6.2) that the geodesic $\gamma_{u}$ with $\gamma_{u}(0)=p$ and $\gamma_{u}^{\prime}(0)=u$ satisfies

$$
\gamma_{u}^{\mathrm{iv}}(s)=-\lambda_{0} h\left(T_{\gamma}, T_{\gamma}\right)+\left(\bar{\nabla}^{2} h\right)\left(T_{\gamma}^{4}\right) .
$$

Differentiating (6.3) and applying (2.11) and (6.2) yields

$$
\gamma_{u}^{v}(s)=\lambda_{0}^{2} T_{\gamma}-A_{\left(\bar{\nabla}^{2} h\right)\left(T_{\gamma}^{4}\right)} T_{\gamma}-\lambda_{0}(\bar{\nabla} h)\left(T_{\gamma}^{3}\right)+\left(\bar{\nabla}^{3} h\right)\left(T_{\gamma}^{5}\right) .
$$

Since $M$ is in contact of order $k \geqslant 5$, we have $\gamma_{u}^{v}(0)=\beta_{u}^{v}(0)$. Because $\beta_{u}^{v}(0)$ is contained in the linear subspace $E(p, u)$ spanned by $u$ and $T_{u}^{\perp}(M)$, (6.4) implies that $u$ is an eigenvector of $A_{\left(\bar{\nabla}^{2} h\right)\left(u^{4}\right)}$, say $A_{\left(\bar{\nabla}^{2} h\right)\left(u^{2}\right)} u=\lambda_{2} u$. So, we obtain Lemma 6.2 for $k=5$.

In order to prove Lemma 6.2 for any $k>5$ by induction, let us assume that $M$ is in contact of order $\ell(\ell>5)$ and Lemma 6.2 is true for $k<\ell$. From our assumption we have

$$
\begin{gathered}
A_{h(u, u)} u=\lambda_{0} u, \quad A_{(\bar{\nabla} h)\left(u^{3}\right)} u=0, \\
A_{\left(\bar{\nabla}^{j} h\right)\left(u^{j+2}\right)} u=\lambda_{j} u, \quad \text { for } j=2, \ldots, \ell-4, \\
\gamma_{u}^{\ell}(0)=\beta_{u}^{\ell}(0) .
\end{gathered}
$$

By differentiating (6.4) and by applying (2.7), (6.5) and (6.6), we find

$$
\gamma_{u}^{\mathrm{vi}}(s)=f_{6} T_{\gamma}-A_{\left(\bar{\nabla}^{3} h\right)\left(T_{\gamma}^{5}\right)} T_{\gamma}+g_{0,6} h\left(T_{\gamma}, T_{\gamma}\right)-\lambda_{0}\left(\bar{\nabla}^{2} h\right)\left(T_{\gamma}^{4}\right)+\left(\bar{\nabla}^{4} h\right)\left(T_{\gamma}^{6}\right),
$$

where

$$
f_{6}=-\lambda_{2}^{\prime}, \quad \lambda_{2}^{\prime}=T_{\gamma}\left(\lambda_{2}\right), \quad g_{0,6}=\lambda_{0}^{2}-\lambda_{2},
$$

and $\lambda_{j}(s)=\lambda_{j}\left(\gamma_{u}^{\prime}(s)\right)$ is the restriction of $\lambda_{j}$ along the unit tangent vector field $T_{\gamma}$ of $\gamma_{u}$. Continuing such procedures $\ell-6$ times and applying (6.6), we obtain

$$
\begin{aligned}
& \gamma_{u}^{\ell-1}(s)=\left(f_{\ell-1}-\lambda_{\ell-4}\right) T_{\gamma}+g_{0, \ell-1} h\left(T_{\gamma}, T_{\gamma}\right)+\cdots \\
& \quad+g_{\ell-7, \ell-1}\left(\bar{\nabla}^{\ell-7} h\right)\left(T_{\gamma}^{\ell-5}\right)-\lambda_{0}\left(\bar{\nabla}^{\ell-5} h\right)\left(T_{\gamma}^{\ell-3}\right)+\left(\bar{\nabla}^{\ell-3} h\right)\left(T_{\gamma}^{\ell-1}\right),
\end{aligned}
$$

where

$$
\begin{aligned}
f_{\ell-1} & =f_{\ell}\left(\lambda_{0}, \lambda_{2}, \ldots, \lambda_{\ell-5}\right), \\
g_{t, \ell-1} & =g_{t, \ell-1}\left(\lambda_{0}, \lambda_{2}, \ldots, \lambda_{\ell-5-t}\right), \quad t=0, \ldots, \ell-7,
\end{aligned}
$$

are functions depending on the $\lambda_{j}^{\prime}$ and their derivatives with respect to $T_{\gamma}$. From (6.5), (6.6) and (6.10), we find

$$
\begin{aligned}
\gamma_{u}^{\ell}(s)=f_{\ell} T_{\gamma}-A_{\left(\bar{\nabla}^{\ell-3} h\right)\left(T_{\gamma}^{\ell-1}\right)} T_{\gamma}+g_{0, \ell} h\left(T_{\gamma}, T_{\gamma}\right)+\cdots & \\
& +g_{\ell-6, \ell}\left(\bar{\nabla}^{\ell-6} h\right)\left(T_{\gamma}^{\ell-4}\right)-\lambda_{0}\left(\bar{\nabla}^{\ell-4} h\right)\left(T_{\gamma}^{\ell-2}\right)+\left(\bar{\nabla}^{\ell-2} h\right)\left(T_{\gamma}^{\ell}\right)
\end{aligned}
$$


where

$$
\left.\begin{array}{rl}
f_{\ell} & =\left(f_{\ell-1}-\lambda_{\ell-4}\right)^{\prime}+\lambda_{0} \lambda_{\ell-5}-\sum_{t=0}^{\ell-7} \lambda_{t} g_{t, \ell-1}, \\
g_{0, \ell} & =f_{\ell-1}-\lambda_{\ell-4}+g_{0, \ell-1}^{\prime}, \\
g_{t, \ell} & =g_{t-1, \ell-1}+g_{t, \ell-1}^{\prime}, \quad t=1, \ldots, \ell-7, \\
g_{\ell-6, \ell} & =g_{\ell-7, \ell-1} .
\end{array}\right\}
$$

Using (6.11), (6.12) and the fact that $\beta_{u}^{\ell}(0)$ lies in $E(p, u)$, we conclude that $u$ is an eigenvector of $A_{\left(\bar{\nabla}^{\ell-3} h\right)\left(u^{\ell-1}\right)}$. Thus, we obtain Lemma 6.2 for $k=\ell$ as well. So, we have proved Lemma 6.2 by induction.

Next, let us prove the converse of Lemma 6.2.

Lemma 6.3. Let $M$ be a submanifold in a Euclidean space. If each $u \in U M$ is an eigenvector of $A_{\left(\bar{\nabla}^{j} h\right)\left(u^{j+2}\right)}$ for $j=0, \ldots, k-3$, then $M$ is in contact of order $k$.

Proof of Lemma 6.3. We already have this lemma for $k=3,4$ according to Lemmas 3.2 and 3.4 and Theorem 4.1. Now we shall prove this lemma for $k=5$. In order to do so, let us assume

$$
A_{h(u, u)} u=\lambda_{0} u, A_{(\bar{\nabla} h)\left(u^{3}\right)} u=0, \quad A_{\left(\bar{\nabla}^{2} h\right)\left(u^{4}\right)} u=\lambda_{2} u,
$$

where $\lambda_{0}$ is constant. We only need to show that $\gamma_{u}^{5}(0)=\beta_{u}^{5}(0)$.

From (4.5) and (6.13) we have

$$
\begin{aligned}
\beta_{u}^{\mathrm{iv}}(s)=\nabla_{T}^{3} T-3 A_{h\left(T, \nabla_{T} T\right)} T-\lambda_{0} \nabla_{T} T-\lambda_{0} h(T, T)+4 h\left(T, \nabla_{T}^{2} T\right) \\
+3 h\left(\nabla_{T} T, \nabla_{T} T\right)+6(\bar{\nabla} h)\left(T, T, \nabla_{T} T\right)+\left(\bar{\nabla}^{2} h\right)\left(T^{4}\right),
\end{aligned}
$$

where $T=T_{\beta}$. Hence, by differentiating (6.14), we find

$$
\begin{aligned}
& \beta_{u}^{v}(s)=\nabla_{T}^{4} T-\lambda_{0} \nabla_{T}^{2} T+\lambda_{0}^{2} T-\lambda_{2} T-3 \nabla_{T}\left(A_{h\left(T, \nabla_{T} T\right)} T\right) \\
& -6 A_{(\bar{\nabla} h)\left(T, T, \nabla_{T} T\right)} T-4 A_{h\left(T, \nabla_{T}^{2} T\right)} T-3 A_{h\left(\nabla_{T} T, \nabla_{T} T\right)} T+h\left(T, \nabla_{T}^{3} T\right) \\
& \quad-3 h\left(A_{h\left(T, \nabla_{T} T\right)} T, T\right)-\lambda_{0} h\left(T, \nabla_{T} T\right)+D_{T}\left(\left(\bar{\nabla}^{2} h\right)\left(T^{4}\right)\right)-\lambda_{0} D_{T} h(T, T) \\
& \quad+6 D_{T}\left((\bar{\nabla} h)\left(T, T, \nabla_{T} T\right)\right)+4 D_{T} h\left(T, \nabla_{T}^{2} T\right)+3 D_{T} h\left(\nabla_{T} T, \nabla_{T} T\right)
\end{aligned}
$$

where $\lambda_{j}(s)=\lambda_{j}\left(\beta_{u}^{\prime}(s)\right)$ is the restriction of $\lambda_{j}$ along $\beta_{u}^{\prime}$. Using (2.6) and (2.7), (6.15) can be restated as

$$
\begin{aligned}
\beta_{u}^{v}(s) & =\nabla_{T}^{4} T+\left(\lambda_{0}^{2}-\lambda_{2}\right) T-\lambda_{0}\left(\bar{\nabla}_{T} h\right)(T, T)+\left(\bar{\nabla}^{3} h\right)\left(T^{5}\right)+\phi_{5}, \\
\phi_{5} & =-\lambda_{0} \nabla_{T}^{2} T-3\left(\nabla_{T} A_{h\left(T, \nabla_{T} T\right)}\right) T-3 A_{h\left(T, \nabla_{T} T\right)}\left(\nabla_{T} T\right)+\cdots,
\end{aligned}
$$

where each term of $\phi_{5}$ is a vector bundle-valued tensor involving at least one of $\nabla_{T} T$, $\nabla_{T}^{2} T, \nabla_{T}^{3} T$. 
On the other hand, from the proof of Theorem 4.1, we have

(i) $\nabla_{u} T_{\beta}=\nabla_{u} \nabla_{T_{\beta}} T_{\beta}=0$;

(ii) $\nabla_{u} \nabla_{T_{\beta}}^{2} T_{\beta}=0$, whenever $M$ is constant isotropic.

Using (6.17), (i) and (ii), we get $\phi_{5}(0)=0$. Hence, (6.16) reduces to

$$
\beta_{u}^{v}(0)=\nabla_{u} \nabla_{T_{\beta}}^{3} T_{\beta}+\left(\lambda_{0}^{2}-\lambda_{2}\right) u-\lambda_{0}(\bar{\nabla} h)\left(u^{3}\right)+\left(\bar{\nabla}^{3} h\right)\left(u^{5}\right) .
$$

Since $\beta_{u}^{v}(0)$ lies in the linear subspace $E(p, u),(6.18)$ implies that

$$
\left\langle\nabla_{u} \nabla_{T_{\beta}}^{3} T_{\beta}, v\right\rangle=0
$$

for $v \in T_{p} M$ perpendicular to $u$. On the other hand, by (i) and (ii) we also have

$$
\begin{aligned}
\left\langle\nabla_{u} \nabla_{T_{\beta}}^{3} T_{\beta}, u\right\rangle & =u\left\langle\nabla_{T_{\beta}}^{3} T_{\beta}, T_{\beta}\right\rangle \\
& =u\left\{T_{\beta}\left\langle\nabla_{T_{\beta}}^{2} T_{\beta}, T_{\beta}\right\rangle-\left\langle\nabla_{T_{\beta}}^{2} T_{\beta}, \nabla_{T_{\beta}} T_{\beta}\right\rangle\right\} \\
& =\frac{1}{2} u\left\{T_{\beta}^{3}\left\langle T_{\beta}, T_{\beta}\right\rangle-3 T_{\beta}\left\langle\nabla_{T_{\beta}} T_{\beta}, \nabla_{T_{\beta}} T_{\beta}\right\rangle\right\} \\
& =-3\left\langle\nabla_{u} \nabla_{T_{\beta}}^{2} T_{\beta}, \nabla_{u} T_{\beta}\right\rangle-3\left\langle\nabla_{u} \nabla_{T_{\beta}} T_{\beta}, \nabla_{u} \nabla_{T_{\beta}} T_{\beta}\right\rangle=0 .
\end{aligned}
$$

Combining this with (6.19) together with (i) and (ii) gives

$$
\nabla_{u} \nabla_{T_{\beta}}^{\ell} T_{\beta}=0, \quad \ell=0,1,2,3 .
$$

Therefore, we have $\gamma_{u}^{v}(0)=\beta_{u}^{v}(0)$ by applying (6.4), (6.18) and (6.21). Hence, we have proved Lemma 6.3 for $k=5$.

Next, we will prove Lemma 6.3 for general $k$ by induction. In order to do so let us assume that, for any given $\ell>5$, Lemma 6.3 is true for $k<\ell$ and we also have

$$
A_{\left(\bar{\nabla}^{j} h\right)\left(u^{j+2}\right)} u=\lambda_{j} u, \quad j=0, \ldots, \ell-4 .
$$

By differentiating (6.16) with respect to $T=T_{\beta}$ and by applying (2.11) and (6.22), we find

$$
\beta_{u}^{\mathrm{vi}}(s)=\nabla_{T}^{5} T-\left(\lambda_{2}^{\prime}+\lambda_{3}\right) T+\left(\lambda_{0}^{2}-\lambda_{2}\right) h(T, T)-\lambda_{0}\left(\bar{\nabla}_{T}^{2} h\right)(T, T)+\left(\bar{\nabla}^{4} h\right)\left(T^{6}\right)+\phi_{6},
$$

where $\phi_{6}$ is a function such that each term of $\phi_{6}$ is expressed in terms of a vector bundle-valued tensor involving at least one of $\nabla_{T} T, \nabla_{T}^{2} T, \nabla_{T}^{3} T, \nabla_{T}^{4} T$. Notice that all of the coefficients of $T,\left(\bar{\nabla}_{T} h\right)(T, T),\left(\bar{\nabla}_{T}^{2} h\right)(T, T)$ and $\left(\bar{\nabla}_{T}^{4} h\right)(T, T)$ in Equation (6.23) are exactly those coefficients in $\gamma_{u}^{\mathrm{vi}}(0)$ given in (6.8).

Repeating such a procedure $\ell-6$ times yields

$$
\begin{aligned}
\beta_{u}^{t}(s)=\nabla_{T}^{t-1} T & +\left(f_{t}-\lambda_{t-3}\right) T+g_{0, t} h(T, T)+\cdots \\
& +g_{t-6, t}\left(\bar{\nabla}^{t-6} h\right)\left(T^{t-4}\right)-\lambda_{0}\left(\bar{\nabla}^{t-4} h\right)\left(T^{t-2}\right)+\left(\bar{\nabla}^{t-2} h\right)\left(T^{t}\right)+\phi_{t}
\end{aligned}
$$


for $t=6, \ldots, \ell$, where $f_{t}, g_{0, t}, \ldots, g_{t-6, t}$ are defined inductively as in (6.12) and each term of $\phi_{t}$ involves at least one of $\nabla_{T} T, \ldots, \nabla_{T}^{t-2} T$. Since $\beta_{u}^{t}(0), t=6, \ldots, \ell$, lie in $E(p, u)$, (6.24) implies that

$$
\left\langle\nabla_{u} \nabla_{T}^{t-2} T, v\right\rangle=0, \quad t=5, \ldots, \ell,
$$

for each $v \in T_{p} M$ perpendicular to $u$.

Next, we claim that (6.25) together with (i) and (ii) implies that

$$
\nabla_{u} \nabla_{T}^{t-2} T=0, \quad t=2, \ldots, \ell .
$$

We can prove this claim inductively as follows: conditions (i) and (ii) imply that (6.26) holds for $\ell=2,3,4$. Let us assume that $\ell \geqslant 5$ and

$$
\nabla_{u} \nabla_{T}^{t-2} T=0, \quad t=2, \ldots, q,
$$

holds for some integer $q \in[4, \ell-1]$. From these we find

$$
\begin{aligned}
\left\langle\nabla_{u} \nabla_{T}^{q-1} T, u\right\rangle & =u\left\langle\nabla_{T}^{q-1} T, T\right\rangle=u\left(T\left\langle\nabla_{T}^{q-2} T, T\right\rangle\right) \\
& =u\left(T^{2}\left\langle\nabla_{T}^{q-3} T, T\right\rangle\right)=\cdots=u\left(T^{q-1}\langle T, T\rangle\right)=0 .
\end{aligned}
$$

Combining this with (6.25) gives $\nabla_{u} \nabla_{T}^{q-1} T=0$. Thus, we obtain (6.26) by induction. Applying (6.24) and (6.26) we find

$$
\begin{aligned}
& \beta_{u}^{\ell}(0)=\left(f_{\ell}-\lambda_{\ell-3}\right) u+g_{0, \ell} h(u, u)+\cdots \\
& +g_{\ell-6, \ell}\left(\bar{\nabla}^{\ell-6} h\right)\left(u^{\ell-4}\right)-\lambda_{0}\left(\bar{\nabla}^{\ell-4} h\right)\left(u^{\ell-2}\right)+\left(\bar{\nabla}^{\ell-2} h\right)\left(u^{\ell}\right) .
\end{aligned}
$$

By comparing (6.11) and (6.29) and by applying (6.22), we have $\gamma_{u}^{\ell}(0)=\beta_{u}^{\ell}(0)$, which implies that $M$ is in contact of order $\ell$. This proves Lemma 6.3 .

Now, Theorem 6.1 follows immediately from Lemmas 6.2 and 6.3.

Theorem 6.1 immediately implies the following.

Corollary 6.4. Every isotropic submanifold with parallel second fundamental form in a Euclidean space satisfies $\mathfrak{c}_{\#}(M)=\infty$.

Remark 6.5. Not every Euclidean submanifold $M$ with parallel second fundamental form satisfies $\mathfrak{c}_{\#}(M)=\infty$. For instance, a circular cylinder $\mathbb{R} \times S^{1}$ in $\mathbb{E}^{3}$ has parallel second fundamental form, but its contact number is 2 , not $\infty$.

We apply Theorem 6.1 to show that the following torus has contact number 4 .

Example 6.6. Consider the isometric immersion $\tau_{a}: \mathbb{E}^{2} \rightarrow \mathbb{E}^{6}$ defined by

$$
\begin{aligned}
\tau_{a}(u, v)=\frac{2}{\sqrt{6} a}\left(\cos \frac{a u}{\sqrt{2}} \cos \right. & \frac{\sqrt{3} a v}{\sqrt{2}}, \cos \frac{a u}{\sqrt{2}} \sin \frac{\sqrt{3} a v}{\sqrt{2}}, \frac{1}{\sqrt{2}} \cos \sqrt{2} a u \\
& \left.\sin \frac{a u}{\sqrt{2}} \cos \frac{\sqrt{3} a v}{\sqrt{2}}, \sin \frac{a u}{\sqrt{2}} \sin \frac{\sqrt{3} a v}{\sqrt{2}}, \frac{1}{\sqrt{2}} \sin \sqrt{2} a u\right) .
\end{aligned}
$$


Then $\tau_{a}$ induces an isometric embedding $\hat{\tau}_{a}: T_{a}^{2} \rightarrow \mathbb{E}^{6}$ of a flat torus $T_{a}^{2}$ into $\mathbb{E}^{6}$ with contact number 4 , which can be seen as follows.

Let $e_{1}=\partial / \partial u$ and $e_{2}=\partial / \partial v$. Then we have

$$
g=\mathrm{d} u^{2}+\mathrm{d} v^{2}, \quad \omega_{1}^{2}=0 .
$$

We put

$$
\begin{aligned}
& e_{3}=-a \phi, \\
& e_{4}=\frac{-1}{\sqrt{3}}\left(\cos \frac{a u}{\sqrt{2}} \cos \frac{\sqrt{3} a v}{\sqrt{2}}, \cos \frac{a u}{\sqrt{2}} \sin \frac{\sqrt{3} a v}{\sqrt{2}},-\sqrt{2} \cos \sqrt{2} a u,\right. \\
& \left.\cos \frac{a u}{\sqrt{2}} \sin \frac{\sqrt{3} a v}{\sqrt{2}}, \sin \frac{a u}{\sqrt{2}} \sin \frac{\sqrt{3} a v}{\sqrt{2}},-\sqrt{2} \sin \sqrt{2} a u\right), \\
& e_{5}=\left(\sin \frac{a u}{\sqrt{2}} \sin \frac{\sqrt{3} a v}{\sqrt{2}},-\cos \frac{a u}{\sqrt{2}} \sin \frac{\sqrt{3} a v}{\sqrt{2}}, 0\right. \text {, } \\
& \left.-\cos \frac{a u}{\sqrt{2}} \sin \frac{\sqrt{3} a v}{\sqrt{2}}, \cos \frac{a u}{\sqrt{2}} \cos \frac{\sqrt{3} a v}{\sqrt{2}}, 0\right), \\
& e_{6}=\sqrt{\frac{2}{3}}\left(\cos \frac{a u}{\sqrt{2}} \sin \frac{\sqrt{3} a v}{\sqrt{2}}, \sin \frac{a u}{\sqrt{2}} \sin \frac{\sqrt{3} a v}{\sqrt{2}}, \frac{-1}{\sqrt{2}} \sin \sqrt{2} a u,\right. \\
& \left.\left.-\cos \frac{a u}{\sqrt{2}} \cos \frac{\sqrt{3} a v}{\sqrt{2}},-\cos \frac{a u}{\sqrt{2}} \sin \frac{\sqrt{3} a v}{\sqrt{2}}, \frac{1}{\sqrt{2}} \cos \sqrt{2} a u\right) .\right)
\end{aligned}
$$

The connection forms of $T_{a}^{2}$ in $\mathbb{E}^{6}$ are given by

$$
\left(\begin{array}{cccccc}
0 & 0 & a \omega^{1} & -\frac{a}{\sqrt{2}} \omega^{1} & \frac{a}{\sqrt{2}} \omega^{2} & 0 \\
0 & 0 & a \omega^{2} & \frac{a}{\sqrt{2}} \omega^{2} & \frac{a}{\sqrt{2}} \omega^{1} & 0 \\
-a \omega^{2} & -a \omega^{2} & 0 & 0 & 0 & 0 \\
\frac{a}{\sqrt{2}} \omega^{1} & -\frac{a}{\sqrt{2}} \omega^{2} & 0 & 0 & 0 & a \omega^{1} \\
-\frac{a}{\sqrt{2}} \omega^{2} & -\frac{a}{\sqrt{2}} \omega^{1} & 0 & 0 & 0 & a \omega^{2} \\
0 & 0 & 0 & -a \omega^{1} & -a \omega^{2} & 0
\end{array}\right),
$$

which implies that

$$
h\left(e_{\theta}, e_{\theta}\right)=e_{3}-\frac{1}{\sqrt{2}} \cos 2 \theta e_{4}+\frac{1}{\sqrt{2}} \sin 2 \theta e_{5},
$$

where $e_{\theta}=\cos \theta e_{1}+\sin \theta e_{2}$. Thus, $\hat{\tau}_{a}$ is an isotropic immersion. After applying (2.6), (2.11), (6.31) and (6.33) we get

$$
(\bar{\nabla} h)\left(e_{\theta}^{3}\right)=-\frac{a \cos 3 \theta}{\sqrt{2}} e_{6}, \quad\left(\bar{\nabla}^{2} h\right)\left(e_{\theta}^{4}\right)=\frac{a^{2} \cos 3 \theta}{\sqrt{2}}\left(\cos \theta e_{4}+\sin \theta e_{5}\right) .
$$


It follows from (6.33) and (6.35) that

$$
A_{(\bar{\nabla} h)\left(e_{\theta}^{3}\right)} e_{\theta}=0, \quad A_{\left(\bar{\nabla}^{2} h\right)\left(e_{\theta}^{4}\right)} e_{\theta}=\frac{1}{2} a^{3} \cos 3 \theta\left(\sin 2 \theta e_{2}-\cos 2 \theta e_{1}\right) .
$$

Thus, by applying Theorem 6.1 , we obtain $\mathfrak{c}_{\#}\left(T_{a}^{2}\right)=4$.

Remark 6.7. There exist many Euclidean submanifolds whose contact numbers are natural numbers greater than 4 . Here we provide such an example.

Example 6.8. Consider a map $\psi: \mathbb{E}^{2} \rightarrow \mathbb{E}^{8}$ defined by

$$
\begin{aligned}
& \psi(x, y) \\
& =\frac{1}{2}\left(\cos \left(\sqrt{\frac{1}{2}(2+\sqrt{2})} x+\sqrt{\frac{1}{2}(2-\sqrt{2})} y\right), \cos \left(\sqrt{\frac{1}{2}(2+\sqrt{2})} a x-\sqrt{\frac{1}{2}(2-\sqrt{2})} y\right)\right. \\
& \sin \left(\sqrt{\frac{1}{2}(2+\sqrt{2})} x+\sqrt{\frac{1}{2}(2-\sqrt{2})} y\right), \sin \left(\sqrt{\frac{1}{2}(2+\sqrt{2})} x-\sqrt{\frac{1}{2}(2-\sqrt{2})} a y\right) \\
& \cos \left(\sqrt{\frac{1}{2}(2-\sqrt{2})} x+\sqrt{\frac{1}{2}(2+\sqrt{2})} y\right), \cos \left(\sqrt{\frac{1}{2}(2-\sqrt{2})} x-\sqrt{\frac{1}{2}(2+\sqrt{2})} a y\right) \\
& \left.\sin \left(\sqrt{\frac{1}{2}(2-\sqrt{2})} x+\sqrt{\frac{1}{2}(2+\sqrt{2})} a y\right), \sin \left(\sqrt{\frac{1}{2}(2-\sqrt{2})} x-\sqrt{\frac{1}{2}(2+\sqrt{2})} y\right)\right) .
\end{aligned}
$$

It is easy to verify that $\psi$ is an isometric immersion. Moreover, a direct long computation shows that its shape operator satisfies

$$
\left.\begin{array}{c}
A_{h\left(e_{\theta}, e_{\theta}\right)} e_{\theta}=\frac{3}{2} e_{\theta}, \quad A_{\bar{\nabla} h\left(e_{\theta}^{3}\right)} e_{\theta}=0, \quad A_{\bar{\nabla}^{2} h\left(e_{\theta}^{4}\right)} e_{\theta}=-\frac{1}{4} e_{\theta}, \\
A_{\bar{\nabla}^{3} h\left(e_{\theta}^{5}\right)} e_{\theta}=0, \quad A_{\bar{\nabla}^{4} h\left(e_{\theta}^{6}\right)} e_{\theta}=\frac{1}{4} e_{\theta}-\frac{1}{8}\left((\cos 7 t) e_{1}-(\sin 7 t) e_{2}\right)
\end{array}\right\}
$$

for any $e_{\theta}=\cos \theta e_{1}+\sin \theta e_{2}$. Hence, this surface has contact number 6 according to Theorem 6.1.

\section{Surfaces in $\mathbb{E}^{6}$ with $\mathfrak{c}_{\#}(M) \geqslant 4$}

A submanifold $M$ is called pseudo-umbilical if its shape operator $A_{\boldsymbol{H}}$ at the mean curvature vector is proportional to the identity map.

Theorem 7.1. Let $M$ be a surface in $\mathbb{E}^{6}$ with constant mean curvature or constant Gauss curvature. If $\mathfrak{c}_{\#}(M) \geqslant 4$, then either $\mathfrak{c}_{\#}(M)=\infty$ or $\mathfrak{c}_{\#}(M)=4$ holds. Moreover, we have the following.

(1) If $\mathfrak{c}_{\#}(M)=\infty, M$ is one of the following three surfaces.

(a) An open portion of a 2-plane.

(b) An open portion of an ordinary 2-sphere lying in a 3-plane of $\mathbb{E}^{6}$.

(c) An open portion of the Veronese surface $S^{2}(\sqrt{3} / a)$ contained in $S^{4}(1 / a)$ which lies in a hyperplane of $\mathbb{E}^{6}$.

(2) If $\mathfrak{c}_{\#}(M)=4$, then $M$ is one of the following two surfaces. 
(a) $M$ is an open portion of a flat torus $T_{a}^{2}$ for some $a>0$ and its immersion is congruent to

$$
\begin{array}{r}
\varphi(u, v)=\frac{2}{\sqrt{6} a}\left(\cos \frac{a u}{\sqrt{2}} \cos \frac{\sqrt{3} a v}{\sqrt{2}}, \cos \frac{a u}{\sqrt{2}} \sin \frac{\sqrt{3} a v}{\sqrt{2}}, \frac{1}{\sqrt{2}} \cos \sqrt{2} a u,\right. \\
\left.\sin \frac{a u}{\sqrt{2}} \cos \frac{\sqrt{3} a v}{\sqrt{2}}, \sin \frac{a u}{\sqrt{2}} \sin \frac{\sqrt{3} a v}{\sqrt{2}}, \frac{1}{\sqrt{2}} \sin \sqrt{2} a u\right) .
\end{array}
$$

(b) $M$ is an open portion of $S^{2}(\sqrt{3} / a)$ immersed linearly fully in $\mathbb{E}^{6}$ as a pseudoumbilical surface with non-parallel mean curvature vector.

Proof. Let $M$ be a surface in $\mathbb{E}^{6}$ with constant mean curvature or constant Gauss curvature. Assume that $\mathfrak{c}_{\#}(M) \geqslant 4$ holds. Then $M$ is constant isotropic by Theorem 4.1.

If $M$ is minimal in $\mathbb{E}^{6}$, then $M$ is totally geodesic (cf. $[\mathbf{5}, \mathbf{2 3}]$ ). Thus, $M$ is an open portion of a 2-plane whose contact number is $\infty$.

From now on, let us assume that $M$ is non-minimal in $\mathbb{E}^{6}$.

Case (i). $M$ is flat. Since $M$ is assumed to be non-minimal, locally we may choose $e_{3}$ so that $\boldsymbol{H}=a e_{3}, a=|\boldsymbol{H}|$. Because $M$ is flat, there exists a local coordinate system $\{x, y\}$ such that the metric tensor of $M$ is given by $g=\mathrm{d} x^{2}+\mathrm{d} y^{2}$. If we put $e_{1}=\partial / \partial x$ and $e_{2}=\partial / \partial y$, we get $\omega_{1}^{2}=0$. Let us choose $e_{4}$ to be a normal vector field such that $h\left(e_{1}, e_{1}\right)=a e_{3}+b e_{4}$. Since $M$ is constant isotropic, we have $\left\langle h\left(e_{1}, e_{2}\right), h\left(e_{1}, e_{1}\right)\right\rangle=$ $\left\langle h\left(e_{1}, e_{2}\right), h\left(e_{2}, e_{2}\right)\right\rangle=0$. Thus, there is a unit vector field $e_{5}$ so that $h\left(e_{1}, e_{2}\right)=\delta e_{5}$ for some function $\delta$. Because $h\left(e_{2}, e_{2}\right)=2 \boldsymbol{H}-h\left(e_{1}, e_{1}\right)$ and $\left|h\left(e_{1}, e_{1}\right)\right|=\left|h\left(e_{2}, e_{2}\right)\right|$, we have $h\left(e_{2}, e_{2}\right)=a e_{3}-b e_{4}$. Hence, we get

$$
h\left(e_{1}, e_{1}\right)=a e_{3}+b e_{4}, \quad h\left(e_{2}, e_{2}\right)=a e_{3}-b e_{4}, \quad h\left(e_{1}, e_{2}\right)=\delta e_{5} .
$$

Since $M$ is constant isotropic, (7.2) implies $\delta= \pm b$. By using the flatness and the equation of Gauss, we get $a^{2}=2 b^{2}$. Consequently, we may choose an orthonormal frame $e_{1}, \ldots, e_{6}$ so that

$$
\left.\begin{array}{c}
h\left(e_{1}, e_{1}\right)=a e_{3}-\frac{a}{\sqrt{2}} e_{4}, \quad h\left(e_{2}, e_{2}\right)=a e_{3}+\frac{a}{\sqrt{2}} e_{4}, \\
h\left(e_{1}, e_{2}\right)=\frac{a}{\sqrt{2}} e_{5}, \quad \omega_{1}^{2}=0,
\end{array}\right\}
$$

where $a=|\boldsymbol{H}|$ is a positive constant due to constant isotropy. From (2.6), (7.2) and (7.3) we find

$$
\begin{aligned}
& \left(\bar{\nabla}_{e_{j}} h\right)\left(e_{1}, e_{1}\right)=a \sum_{r=3}^{6}\left(\omega_{3}^{r}\left(e_{j}\right)-\frac{1}{\sqrt{2}} \omega_{4}^{r}\left(e_{j}\right)\right) e_{r}, \\
& \left(\bar{\nabla}_{e_{j}} h\right)\left(e_{1}, e_{2}\right)=\frac{a}{\sqrt{2}} \sum_{r=3}^{6} \omega_{5}^{r}\left(e_{j}\right) e_{r}, \\
& \left(\bar{\nabla}_{e_{j}} h\right)\left(e_{2}, e_{2}\right)=a \sum_{r=3}^{6}\left(\omega_{3}^{r}\left(e_{j}\right)+\frac{1}{\sqrt{2}} \omega_{4}^{r}\left(e_{j}\right)\right) e_{r}, \quad j=1,2 .
\end{aligned}
$$


Using (7.4)-(7.6) and the equation of Codazzi, we find

$$
\begin{array}{lll}
\omega_{5}^{3}\left(e_{1}\right)=\omega_{3}^{4}\left(e_{2}\right), & \omega_{5}^{4}\left(e_{1}\right)=\sqrt{2} \omega_{3}^{4}\left(e_{2}\right), & \omega_{4}^{5}\left(e_{2}\right)=\sqrt{2} \omega_{3}^{5}\left(e_{2}\right), \\
\omega_{5}^{3}\left(e_{2}\right)=\omega_{4}^{3}\left(e_{1}\right), & \omega_{5}^{4}\left(e_{2}\right)=\sqrt{2} \omega_{3}^{4}\left(e_{1}\right), & \sqrt{2} \omega_{3}^{5}\left(e_{1}\right)=\omega_{5}^{4}\left(e_{1}\right),
\end{array}
$$

which imply that $\omega_{3}^{4}=\omega_{3}^{5}=\omega_{4}^{5}=0$. Moreover, (7.4)-(7.6) and the equation of Codazzi also imply that

$$
\sqrt{2} \omega_{3}^{6}\left(e_{2}\right)-\omega_{4}^{6}\left(e_{2}\right)=\omega_{5}^{6}\left(e_{1}\right), \quad \sqrt{2} \omega_{3}^{6}\left(e_{1}\right)+\omega_{4}^{6}\left(e_{1}\right)=\omega_{5}^{6}\left(e_{2}\right) .
$$

If we put

$$
\omega_{3}^{6}=\mu \omega^{1}+\varphi \omega^{2}, \quad \omega_{4}^{6}=\psi \omega^{1}+\eta \omega^{2}, \quad \omega_{5}^{6}=p \omega^{1}+q \omega^{2},
$$

then (7.7) and (7.8) give

$$
\sqrt{2} \varphi-\eta=p, \quad \sqrt{2} \mu+\psi=q .
$$

By taking the exterior derivatives of $\omega_{3}^{4}, \omega_{3}^{5}, \omega_{3}^{6}, \omega_{4}^{5}, \omega_{4}^{6}, \omega_{5}^{6}$ and applying (7.2), (7.9), $\omega_{3}^{4}=\omega_{3}^{5}=\omega_{4}^{5}=0$ and structure equations, we find

$$
\begin{aligned}
\mu \eta & =\psi \varphi, & \mu q & =\varphi p, & q \psi-p \eta & =a^{2}, \\
\frac{\partial \varphi}{\partial x} & =\frac{\partial \mu}{\partial y}, & \frac{\partial \eta}{\partial x} & =\frac{\partial \psi}{\partial y}, & \frac{\partial q}{\partial x} & =\frac{\partial p}{\partial y} .
\end{aligned}
$$

Case (i) (a). $\eta, q, \varphi \neq 0$. In this case, the first two equations in (7.10) imply that $\mu / \varphi=\psi / \eta=p / q$. So, we get $p \eta-q \psi=0$, which contradicts the third equation in (7.10). So, this case is impossible.

Case (i)(b). $\varphi=0$. In this case, (7.9) and (7.10) imply $\eta=-p$ and $\mu \eta=\mu q=0$. If $\mu \neq 0$, we obtain $\eta=q=0$, which contradicts the third equation in (7.10). So, we must have $\mu=0$. So, we obtain from (7.9) that $\psi=q$. Hence, the third equation in (7.10) yields $p^{2}+q^{2}=a^{2}$. Moreover, from (7.11), we also have

$$
\frac{\partial q}{\partial x}=\frac{\partial p}{\partial y}, \quad \frac{\partial q}{\partial y}=-\frac{\partial p}{\partial x} .
$$

Since $p^{2}+q^{2}=a^{2}$, we may put $p=a \cos \theta(x, y), q=a \sin \theta(x, y)$ for some $\theta$. Hence, $\theta$ is constant due to (7.12). Thus, the connection forms of $M$ are given by

$$
\left(\begin{array}{cccccc}
0 & 0 & a \omega^{1} & -\frac{a}{\sqrt{2}} \omega^{1} & \frac{a}{\sqrt{2}} \omega^{2} & 0 \\
0 & 0 & a \omega^{2} & \frac{a}{\sqrt{2}} \omega^{2} & \frac{a}{\sqrt{2}} \omega^{1} & 0 \\
-a \omega^{2} & -a \omega^{2} & 0 & 0 & 0 & 0 \\
\frac{a}{\sqrt{2}} \omega^{1} & -\frac{a}{\sqrt{2}} \omega^{2} & 0 & 0 & 0 & q \omega^{1}-p \omega^{2} \\
-\frac{a}{\sqrt{2}} \omega^{2} & -\frac{a}{\sqrt{2}} \omega^{1} & 0 & 0 & 0 & p \omega^{1}+q \omega^{2} \\
0 & 0 & 0 & -q \omega^{1}+p \omega^{2} & -p \omega^{1}-q \omega^{2} & 0
\end{array}\right)
$$

for some constants $p, q$. 
Now, let us consider a new field of orthonormal frame $\left\{\bar{e}_{1}, \ldots, \bar{e}_{6}\right\}$ by

$$
\begin{aligned}
& \bar{e}_{1}=\cos \left(\frac{\theta}{3}-\frac{\pi}{6}\right) e_{1}+\sin \left(\frac{\theta}{3}-\frac{\pi}{6}\right) e_{2}, \\
& \bar{e}_{2}=-\sin \left(\frac{\theta}{3}-\frac{\pi}{6}\right) e_{1}+\cos \left(\frac{\theta}{3}-\frac{\pi}{6}\right) e_{2}, \\
& \bar{e}_{3}=e_{3} \\
& \bar{e}_{4}=\cos \left(\frac{2 \theta}{3}-\frac{\pi}{3}\right) e_{4}-\sin \left(\frac{2 \theta}{3}-\frac{\pi}{3}\right) e_{5}, \\
& \bar{e}_{5}=\sin \left(\frac{2 \theta}{3}-\frac{\pi}{3}\right) e_{4}+\cos \left(\frac{2 \theta}{3}-\frac{\pi}{3}\right) e_{5}, \\
& \bar{e}_{6}=e_{6}
\end{aligned}
$$

The connection forms of the surface with respect to this new frame are given by

$$
\left(\begin{array}{cccccc}
0 & 0 & a \omega^{1} & -\frac{a}{\sqrt{2}} \omega^{1} & \frac{a}{\sqrt{2}} \omega^{2} & 0 \\
0 & 0 & a \omega^{2} & \frac{a}{\sqrt{2}} \omega^{2} & \frac{a}{\sqrt{2}} \omega^{1} & 0 \\
-a \omega^{2} & -a \omega^{2} & 0 & 0 & 0 & 0 \\
\frac{a}{\sqrt{2}} \omega^{1} & -\frac{a}{\sqrt{2}} \omega^{2} & 0 & 0 & 0 & a \omega^{1} \\
-\frac{a}{\sqrt{2}} \omega^{2} & -\frac{a}{\sqrt{2}} \omega^{1} & 0 & 0 & 0 & a \omega^{2} \\
0 & 0 & 0 & -a \omega^{1} & -a \omega^{2} & 0
\end{array}\right) .
$$

Since the surface $T_{a}^{2}$ in Example 6.6 and the surface $M$ above are both flat and they share the same connection forms, we conclude from the uniqueness theorem of submanifolds that $M$ is congruent to an open portion of $T_{a}^{2}$ in $\mathbb{E}^{6}$. It is known from $\S 6$ that the contact number of $T_{a}^{2}$ is 4 .

Case (i)(c). $\eta=0$. In this case, (7.10) implies $\psi \varphi=0, q \psi=-a^{2} \neq 0$. Thus, we have $\varphi=0$. Thus, this case reduces to Case (i)(b).

Case (i)(d). $q=0$. In this case, the last two equations in (7.10) imply $\varphi p=0$, $p \eta=a^{2} \neq 0$. Thus, $\varphi=0$. So, this reduces to Case (i)(b) as well.

Case (ii). $M$ is non-flat. Since $M$ is constant isotropic, we may choose $e_{1}, \ldots, e_{6}$ as in Case (i) so that

$$
h\left(e_{1}, e_{1}\right)=a e_{3}+b e_{4}, \quad h\left(e_{2}, e_{2}\right)=a e_{3}-b e_{4}, \quad h\left(e_{1}, e_{2}\right)=b e_{5}
$$

for some functions $a, b$. It follows from constant isotropy and (7.15) that $a^{2}+b^{2}$ is constant. Thus, the hypothesis 'the mean curvature or Gauss curvature is constant' implies that $a, b$ are constant. So, $M$ has constant mean curvature and constant Gauss curvature. Also, since $M$ is assumed to be non-minimal, we have $a \neq 0$. 
Case (ii)(a). $b=0$. In this case, the surface is totally umbilical. Hence, $M$ is an open portion of an ordinary 2 -sphere in $\mathbb{E}^{6}$ which has contact number $\infty$.

Case (ii) (b). $b \neq 0$. In this case, the first normal space $\operatorname{Im} h$ is spanned by $e_{3}, e_{4}, e_{5}$. On the other hand, since $a, b$ are constant, (2.6) and (7.15) imply that

$$
\begin{aligned}
& \left(\bar{\nabla}_{e_{j}} h\right)\left(e_{1}, e_{1}\right)=\sum_{r=3}^{m}\left(a \omega_{3}^{r}\left(e_{j}\right)+b \omega_{4}^{r}\left(e_{j}\right)\right) e_{r}-2 b \omega_{1}^{2}\left(e_{j}\right) e_{5}, \\
& \left(\bar{\nabla}_{e_{j}} h\right)\left(e_{1}, e_{2}\right)=\sum_{r=3}^{m} b \omega_{5}^{r}\left(e_{j}\right) e_{r}+2 b \omega_{1}^{2}\left(e_{j}\right) e_{4}, \\
& \left(\bar{\nabla}_{e_{j}} h\right)\left(e_{2}, e_{2}\right)=\sum_{r=3}^{m}\left(a \omega_{3}^{r}\left(e_{j}\right)-b \omega_{4}^{r}\left(e_{j}\right)\right) e_{r}+2 b \omega_{1}^{2}\left(e_{j}\right) e_{5}, \quad j=1,2 .
\end{aligned}
$$

From (7.16)-(7.18) and the equation of Codazzi, we find

$$
\begin{array}{llrl}
\omega_{5}^{3}\left(e_{1}\right) & =\omega_{4}^{3}\left(e_{2}\right), & b \omega_{5}^{4}\left(e_{1}\right)+2 b \omega_{1}^{2}\left(e_{1}\right)=a \omega_{3}^{4}\left(e_{2}\right), & a \omega_{3}^{5}\left(e_{2}\right)+b \omega_{4}^{5}\left(e_{2}\right)=2 b \omega_{1}^{2}\left(e_{2}\right), \\
\omega_{5}^{3}\left(e_{2}\right)=\omega_{3}^{4}\left(e_{1}\right), & b \omega_{5}^{4}\left(e_{2}\right)+2 b \omega_{1}^{2}\left(e_{2}\right)=a \omega_{3}^{4}\left(e_{1}\right), & a \omega_{3}^{5}\left(e_{1}\right)+2 b \omega_{1}^{2}\left(e_{1}\right)=b \omega_{4}^{5}\left(e_{1}\right),
\end{array}
$$

which imply

$$
\omega_{3}^{4}=\omega_{3}^{5}=0, \quad \omega_{4}^{5}=2 \omega_{1}^{2} .
$$

Moreover, from (7.16)-(7.18) and the equation of Codazzi, we also have

$$
\begin{aligned}
& a \omega_{3}^{6}\left(e_{2}\right)+b \omega_{4}^{6}\left(e_{2}\right)=b \omega_{5}^{6}\left(e_{1}\right), \\
& a \omega_{3}^{6}\left(e_{1}\right)-b \omega_{4}^{6}\left(e_{1}\right)=b \omega_{5}^{6}\left(e_{2}\right) .
\end{aligned}
$$

Case (ii)(b)(1). $D \boldsymbol{H}=0$. In this case, $M$ is immersed as a minimal surface in a hypersphere of $\mathbb{E}^{6}$, because $M$ is pseudo-umbilical with parallel mean curvature vector (see [6]). Since $M$ is non-flat, the Gauss curvature of $M$ is positive (cf. [4]). So, $M$ is immersed as a Veronese surface lying in a hypersphere of a hyperplane in $\mathbb{E}^{6}$ according to Theorem 1.6 of [4]. The Veronese surface has contact number $\infty$.

Case (ii) (b)(2). $D \boldsymbol{H} \neq 0$. In this case, we have $\omega_{3}^{6} \neq 0$. So, by differentiating $\omega_{3}^{4}=0$ and applying (7.15) and (7.19), we get $\omega_{3}^{6} \wedge \omega_{4}^{6}=0$, which implies that

$$
\omega_{4}^{6}=\psi \omega_{3}^{6}
$$

for some function $\psi$. Similarly, by differentiating $\omega_{3}^{5}=0$ and applying (7.15) and (7.19), we get $\omega_{3}^{6} \wedge \omega_{5}^{6}=0$. Thus, we also have

$$
\omega_{5}^{6}=\phi \omega_{3}^{6}
$$

for some function $\phi$. On the other hand, by differentiating $\omega_{4}^{5}=2 \omega_{1}^{2}$, we find

$$
\omega_{4}^{6} \wedge \omega_{5}^{6}=2\left(a^{2}-3 b^{2}\right) \omega^{1} \wedge \omega^{2} .
$$


Combining (7.22), (7.23) and (7.24), we obtain $a^{2}=3 b^{2}$, which implies that $M$ has constant Gauss curvature $K=a^{2} / 3$.

Replacing $e_{4}, e_{5}$ by $-e_{4},-e_{5}$ if necessary, we have

$$
h\left(e_{1}, e_{1}\right)=a e_{3}+\frac{a}{\sqrt{3}} e_{4}, \quad h\left(e_{2}, e_{2}\right)=a e_{3}-\frac{a}{\sqrt{3}} e_{4}, \quad h\left(e_{1}, e_{2}\right)=\frac{a}{\sqrt{3}} e_{5} .
$$

If we put $\omega_{1}^{2}=p \omega^{1}+q \omega^{2}, \omega_{3}^{6}=\lambda \omega^{1}+\mu \omega^{2}$, we obtain from (7.19), (7.22) and (7.23) that

$$
\left.\begin{array}{lll}
\omega_{1}^{2}=p \omega^{1}+q \omega^{2}, & \omega_{3}^{4}=\omega_{3}^{5}=0, & \omega_{3}^{6}=\lambda \omega^{1}+\mu \omega^{2}, \\
\omega_{4}^{5}=2 p \omega^{1}+2 q \omega^{2}, & \omega_{4}^{6}=\psi \lambda \omega^{1}+\psi \mu \omega^{2}, & \omega_{5}^{6}=\phi \lambda \omega^{1}+\phi \mu \omega^{2} .
\end{array}\right\}
$$

Using (7.20), (7.21) and (7.26) we find

$$
\sqrt{3} \mu+\psi \mu=\phi \lambda, \quad \sqrt{3} \lambda-\psi \lambda=\phi \mu
$$

which implies that

$$
\psi=\frac{\sqrt{3}\left(\lambda^{2}-\mu^{2}\right)}{\lambda^{2}+\mu^{2}}, \quad \phi=\frac{2 \sqrt{3} \lambda \mu}{\lambda^{2}+\mu^{2}} .
$$

From (7.16)-(7.18) and (7.26), we find

$$
\left.\begin{array}{ll}
(\bar{\nabla} h)\left(e_{1}, e_{1}, e_{1}\right)=a \lambda\left(1+\frac{\psi}{\sqrt{3}}\right) e_{6}, & (\bar{\nabla} h)\left(e_{1}, e_{1}, e_{2}\right)=\frac{a \phi \lambda}{\sqrt{3}} e_{6}, \\
(\bar{\nabla} h)\left(e_{1}, e_{2}, e_{2}\right)=\frac{a \phi \mu}{\sqrt{3}} e_{6}, & (\bar{\nabla} h)\left(e_{2}, e_{2}, e_{2}\right)=a \mu\left(1-\frac{\psi}{\sqrt{3}}\right) e_{6} .
\end{array}\right\}
$$

Using (2.7), (7.26) and (7.28), we obtain

$$
\left.\begin{array}{l}
h_{1111}=-(\sqrt{3}+\psi) \lambda^{2} E_{3}+\left\{(\sqrt{3}+\psi) e_{1} \lambda+\lambda e_{1} \psi-3 p \lambda \phi\right\} E_{6}, \\
h_{1121}=-\phi \lambda^{2} E_{3}+\left\{\phi e_{1} \lambda+\lambda e_{1} \phi-2 p \phi \mu+(\sqrt{3}+\psi) p \lambda\right\} E_{6}, \\
h_{1221}=-\phi \lambda \mu E_{3}+\left\{\phi e_{1} \mu+\mu e_{1} \phi+2 p \phi \lambda-(\sqrt{3}-\psi) p \mu\right\} E_{6}, \\
h_{2221}=(\psi-\sqrt{3}) \lambda \mu E_{3}+\left\{(\sqrt{3}-\psi) e_{1} \mu-\mu e_{1} \psi+3 p \mu \phi\right\} E_{6}, \\
h_{1112}=-(\sqrt{3}+\psi) \lambda \mu E_{3}+\left\{(\sqrt{3}+\psi) e_{2} \lambda+\lambda e_{2} \psi-3 q \lambda \phi\right\} E_{6}, \\
h_{1122}=-\phi \lambda \mu E_{3}+\left\{\phi e_{2} \lambda+\lambda e_{2} \phi-2 q \phi \mu+(\sqrt{3}+\psi) q \lambda\right\} E_{6}, \\
h_{1222}=-\phi \mu^{2} E_{3}+\left\{\phi e_{2} \mu+\mu e_{2} \phi+2 q \phi \lambda-(\sqrt{3}-\psi) q \mu\right\} E_{6}, \\
h_{2222}=(\psi-\sqrt{3}) \mu^{2} E_{3}+\left\{(\sqrt{3}-\psi) e_{2} \mu-\mu e_{2} \psi+3 q \mu \phi\right\} E_{6},
\end{array}\right\}
$$

where

$$
E_{3}=\frac{a}{\sqrt{3}}\left(e_{3}+\psi e_{4}+\phi e_{5}\right), \quad E_{6}=\frac{a}{\sqrt{3}} e_{6} \quad \text { and } \quad h_{i j k \ell}=\left(\bar{\nabla}^{2} h\right)\left(e_{i}, e_{j}, e_{k}, e_{\ell}\right) .
$$


Thus

$$
\left.\begin{array}{l}
A_{\left(\bar{\nabla}^{2} h\right)\left(e_{1}^{4}\right)} e_{1}=-\frac{1}{3} a^{2} \lambda^{2}(\sqrt{3}+\psi)\left((\sqrt{3}+\psi) e_{1}+\phi e_{2}\right), \\
A_{\left(\bar{\nabla}^{2} h\right)\left(e_{1}^{4}\right)} e_{2}=\frac{1}{3} a^{2} \mu^{2}(\psi-\sqrt{3})\left((\sqrt{3}+\psi) e_{1}+\phi e_{2}\right) .
\end{array}\right\}
$$

If $\mathfrak{c}_{\#}(M) \geqslant 5,(7.31)$ and Theorem 6.1 imply $\psi= \pm \sqrt{3}$. When $\psi=\sqrt{3}$ holds, (7.28) yields $\mu=\phi=0$. Similarly, if $\psi=-\sqrt{3}$ holds, we obtain $\lambda=\phi=0$.

Suppose that $\psi=\sqrt{3}$ and $\mu=\phi=0$ hold. Then (7.30) becomes

$$
\left.\begin{array}{rl}
h_{1111} & =-2 \sqrt{3} \lambda^{2} E_{3}+2 \sqrt{3}\left(e_{1} \lambda\right) E_{6}, \\
h_{1121} & =2 \sqrt{3} p \lambda E_{6}, \\
h_{1221} & =h_{2221}=h_{1222}=h_{2222}=0, \\
h_{1112} & =2 \sqrt{3}\left(e_{2} \lambda\right) E_{6}, \\
h_{1122} & =2 \sqrt{3} q \lambda E_{6} .
\end{array}\right\}
$$

Hence, we may obtain from (7.25) and (7.32) that

$$
A_{\left(\bar{\nabla}^{2} h\right)\left(e_{\theta}^{4}\right)} e_{\theta}=-4 a^{2} \lambda^{2} \cos ^{5} \theta e_{1}, \quad e_{\theta}=\cos \theta e_{1}+\sin \theta e_{2} .
$$

Thus, by Theorem 6.1 , we get $\lambda=0$, which is a contradiction. Similarly, we also obtain a contradiction (given by $\mu=0$ ) if $\psi=-\sqrt{3}$ holds. Consequently, we have $\mathfrak{c}_{\#}(M)=4$ when $M$ has non-parallel mean curvature vector. It is obvious that every surface given in $(2)(b)$ lies fully in $\mathbb{E}^{6}$.

\section{Classification of surfaces of Case (2)(b) in Theorem 7.1}

The following result classifies surfaces of Case (2)(b) in Theorem 7.1.

Theorem 8.1. Let $a>0$ and let $\{\lambda(u, v), \mu(u, v)\}$ be non-trivial solutions of the system of partial differential equations

$$
\begin{gathered}
\lambda_{v}=\left(\mu \cos \left(\frac{a u}{\sqrt{3}}\right)\right)_{u}, \\
\lambda^{2} \mu_{v}+\cos \left(\frac{a u}{\sqrt{3}}\right) \lambda_{u} \mu^{2}-\frac{2 a}{\sqrt{3}} \sin \left(\frac{a u}{\sqrt{3}}\right) \lambda \mu^{2}-2 \lambda \lambda_{v} \mu=\frac{a}{\sqrt{3}} \sin \left(\frac{a u}{\sqrt{3}}\right) \lambda^{3}
\end{gathered}
$$

defined on a simply connected open set $V_{\lambda, \mu}^{a}$. Let $U_{\lambda, \mu}^{a}$ be the open subset of $S^{2}(\sqrt{3} / a)$ with metric $g=\mathrm{d} u^{2}+\cos ^{2}(a u / \sqrt{3}) \mathrm{d} v^{2}$ defined on $V_{\lambda, \mu}^{a}$. Then, up to rigid motions, there exists a unique pseudo-umbilical isometric immersion $\psi_{\lambda, \mu}^{a}: U_{\lambda, \mu}^{a} \rightarrow \mathbb{E}^{6}$ with contact number 4 , constant mean curvature $a$, and whose mean curvature vector satisfies

$$
D_{\partial / \partial u} \boldsymbol{H}=a \lambda \xi, \quad D_{\partial / \partial v} \boldsymbol{H}=a \mu \cos \left(\frac{a u}{\sqrt{3}}\right) \xi,
$$

where $\xi$ is a unit normal vector field orthogonal to the first normal bundle of $\psi_{\lambda, \mu}^{a}$.

Conversely, every surface in $\mathbb{E}^{6}$ with contact number 4 , constant mean curvature, and non-parallel mean curvature vector is given by a pseudo-umbilical immersion of an open portion of $S^{2}(\sqrt{3} / a)$ which is congruent to a $\psi_{\lambda, \mu}^{a}$ obtained in the way described above. 
Proof. Let $S^{2}(\sqrt{3} / a)$ be the 2 -sphere with constant curvature $3 / a^{2}$. We choose a local coordinate system $\{u, v\}$ on $S^{2}(\sqrt{3} / a)$ such that the metric tensor is given by

$$
g=\mathrm{d} u^{2}+\cos ^{2}\left(\frac{a u}{\sqrt{3}}\right) \mathrm{d} v^{2} .
$$

Assume that $\{\lambda, \mu\}$ are non-trivial solutions of system (8.1), (8.2) defined on a simply connected open set $V_{\lambda, \mu}^{a}$. Let $E$ be a four-dimensional Riemannian vector bundle over $U_{\lambda, \mu}^{a}$ and $h$ be an $E$-valued symmetric $(0,2)$-tensor on $U_{\lambda, \mu}^{a}$ defined by

$$
\left.\begin{array}{c}
h\left(\frac{\partial}{\partial u}, \frac{\partial}{\partial u}\right)=a e_{3}+\frac{a}{\sqrt{3}} e_{4}, \quad h\left(\frac{\partial}{\partial u}, \frac{\partial}{\partial v}\right)=\frac{a}{\sqrt{3}} \cos \left(\frac{a u}{\sqrt{3}}\right) e_{5}, \\
h\left(\frac{\partial}{\partial v}, \frac{\partial}{\partial v}\right)=a \cos ^{2}\left(\frac{a u}{\sqrt{3}}\right) e_{3}-\frac{a}{\sqrt{3}} \cos ^{2}\left(\frac{a u}{\sqrt{3}}\right) e_{4},
\end{array}\right\}
$$

where $e_{3}, e_{4}, e_{5}, e_{6}$ are orthonormal cross-sections of $E$. We define a linear connection $D$ on $E$ by

$$
\left.\begin{array}{rl}
D_{\partial / \partial u} e_{3}= & \lambda e_{6}, \\
D_{\partial / \partial v} e_{3}= & \mu \cos \left(\frac{a u}{\sqrt{3}}\right) e_{6}, \\
D_{\partial / \partial u} e_{4}= & \frac{\sqrt{3} \lambda\left(\lambda^{2}-\mu^{2}\right)}{\lambda^{2}+\mu^{2}} e_{6}, \\
D_{\partial / \partial v} e_{4}= & -\frac{2 a}{\sqrt{3}} \sin \left(\frac{a u}{\sqrt{3}}\right) e_{5}+\frac{\sqrt{3} \mu\left(\lambda^{2}-\mu^{2}\right)}{\lambda^{2}+\mu^{2}} \cos \left(\frac{a u}{\sqrt{3}}\right) e_{6}, \\
D_{\partial / \partial u} e_{5}= & \frac{2 \sqrt{3} \lambda^{2} \mu}{\lambda^{2}+\mu^{2}} e_{6}, \\
D_{\partial / \partial v} e_{5}= & \frac{2 a}{\sqrt{3}} \sin \left(\frac{a u}{\sqrt{3}}\right) e_{4}+\frac{2 \sqrt{3} \lambda \mu^{2}}{\lambda^{2}+\mu^{2}} \cos \left(\frac{a u}{\sqrt{3}}\right) e_{6}, \\
D_{\partial / \partial u} e_{6}= & -\lambda e_{3}-\frac{\sqrt{3} \lambda\left(\lambda^{2}-\mu^{2}\right)}{\lambda^{2}+\mu^{2}} e_{4}-\frac{2 \sqrt{3} \lambda^{2} \mu}{\lambda^{2}+\mu^{2}} e_{5}, \\
D_{\partial / \partial v} e_{6}= & -\mu \cos \left(\frac{a u}{\sqrt{3}}\right) e_{3}-\frac{\sqrt{3} \mu\left(\lambda^{2}-\mu^{2}\right)}{\lambda^{2}+\mu^{2}} \cos \left(\frac{a u}{\sqrt{3}}\right) e_{4}-\frac{2 \sqrt{3} \lambda \mu^{2}}{\lambda^{2}+\mu^{2}} \cos \left(\frac{a u}{\sqrt{3}}\right) e_{5},
\end{array}\right\}
$$

For each cross-section $\eta$ of $E$, we define $A_{\eta}$ by $g\left(A_{\eta} X, Y\right)=\langle h(X, Y), \eta\rangle$, where $\langle\cdot, \cdot\rangle$ is the fibre metric. A direct long computation shows that $\left(U_{\lambda, \mu}^{a}, g, E, D, h, A\right)$ satisfies the equations of Gauss, Codazzi and Ricci. Hence, by the existence theorem of submanifolds, there exists an isometric immersion $\psi_{\lambda, \mu}^{a}$ from $\left(U_{\lambda, \mu}^{a}, g\right)$ into $\mathbb{E}^{6}$ whose normal bundle, second fundamental form, shape operator and normal connection are given, respectively, by $E, h, A$ and $D$ (see $[6])$.

It follows from (8.5) and (8.6) that $\psi_{\lambda, \mu}^{a}$ is a pseudo-umbilical immersion with constant mean curvature $a$ and whose mean curvature vector satisfies (8.3) with $\xi=e_{6}$. Clearly, $\xi$ is perpendicular to the first normal space at each point. Also, we know that the contact 
number of $\psi_{\lambda, \mu}^{a}$ is 4 by applying an argument similar to the one given in the proof of Theorem 7.1.

Conversely, let $z: M \rightarrow \mathbb{E}^{6}$ be a surface with constant mean curvature, contact number 4 , and non-parallel mean curvature vector. Then $z$ is constant isotropic and, moreover, according to Theorem 7.1, it is an open portion of $S^{2}(\sqrt{3} / a), a>0$, immersed fully in $\mathbb{E}^{6}$ as a pseudo-umbilical surface with $a$ as its mean curvature. Thus, there exist orthonormal normal vector fields $e_{3}, \ldots, e_{6}$ such that

$$
A_{3}=\left(\begin{array}{cc}
a & 0 \\
0 & a
\end{array}\right), \quad A_{4}=\left(\begin{array}{cc}
b & 0 \\
0 & -b
\end{array}\right), \quad A_{5}=\left(\begin{array}{cc}
c & k \\
k & -c
\end{array}\right), \quad A_{6}=0
$$

for some functions $b, k$ with respect to $e_{1}=\partial / \partial u, e_{2}=\sec (a u / \sqrt{3}) \partial / \partial v$. Because $M$ is constant isotropic, we get $c=0$ and $b= \pm k$. Hence, (8.7) reduces to

$$
A_{3}=\left(\begin{array}{cc}
a & 0 \\
0 & a
\end{array}\right), \quad A_{4}=\left(\begin{array}{cc}
b & 0 \\
0 & -b
\end{array}\right), \quad A_{5}=\left(\begin{array}{ll}
0 & b \\
b & 0
\end{array}\right), \quad A_{6}=0
$$

after replacing $e_{5}$ by $-e_{5}$ if necessary. Since $M$ has constant Gauss curvature $3 / a^{2}$, we obtain from (8.8) that $b=a / \sqrt{3}$. Therefore, we get

$$
h\left(e_{1}, e_{1}\right)=a e_{3}+\frac{a}{\sqrt{3}} e_{4}, \quad h\left(e_{2}, e_{2}\right)=a e_{3}-\frac{a}{\sqrt{3}} e_{4}, \quad h\left(e_{1}, e_{2}\right)=\frac{a}{\sqrt{3}} e_{5} .
$$

By applying (2.6) and (8.9), we find

$$
\left.\begin{array}{l}
\left(\bar{\nabla}_{e_{j}} h\right)\left(e_{1}, e_{1}\right)=\sum_{r=3}^{6}\left(a \omega_{3}^{r}\left(e_{j}\right)+\frac{a}{\sqrt{3}} \omega_{4}^{r}\left(e_{j}\right)\right) e_{r}-\frac{2 a}{\sqrt{3}} \omega_{1}^{2}\left(e_{j}\right) e_{5}, \\
\left(\bar{\nabla}_{e_{j}} h\right)\left(e_{1}, e_{2}\right)=\sum_{r=3}^{6} \frac{a}{\sqrt{3}} \omega_{5}^{r}\left(e_{j}\right) e_{r}+\frac{2 a}{\sqrt{3}} \omega_{1}^{2}\left(e_{j}\right) e_{4}, \\
\left(\bar{\nabla}_{e_{j}} h\right)\left(e_{2}, e_{2}\right)=\sum_{r=3}^{6}\left(a \omega_{3}^{r}\left(e_{j}\right)-\frac{a}{\sqrt{3}} \omega_{4}^{r}\left(e_{j}\right)\right) e_{r}+\frac{2 a}{\sqrt{3}} \omega_{1}^{2}\left(e_{j}\right) e_{5}, \quad j=1,2 .
\end{array}\right\}
$$

We obtain from (8.10) that

$$
\omega_{3}^{4}=\omega_{3}^{5}=0, \quad \omega_{4}^{5}=2 \omega_{1}^{2}, \quad \omega_{1}^{2}=\frac{a}{\sqrt{3}} \tan \left(\frac{a u}{\sqrt{3}}\right) .
$$

Moreover, using (8.10) and the equation of Codazzi, we have

$$
\begin{aligned}
& \sqrt{3} \omega_{3}^{6}\left(e_{2}\right)+\omega_{4}^{6}\left(e_{2}\right)=\omega_{5}^{6}\left(e_{1}\right), \\
& \sqrt{3} \omega_{3}^{6}\left(e_{1}\right)-\omega_{4}^{6}\left(e_{1}\right)=\omega_{5}^{6}\left(e_{2}\right) .
\end{aligned}
$$

Since the mean curvature vector is non-parallel, we get $\omega_{3}^{6} \neq 0$. Thus, by differentiating $\omega_{3}^{4}=0$ and applying (8.9) and (8.11), we discover that $\omega_{3}^{6} \wedge \omega_{4}^{6}=0$. So, we have

$$
\omega_{4}^{6}=\psi \omega_{3}^{6}
$$


for some function $\psi$. Similarly, by differentiating $\omega_{3}^{5}=0$ and applying (8.9) and (8.11), we get $\omega_{3}^{6} \wedge \omega_{5}^{6}=0$. So, we also have

$$
\omega_{5}^{6}=\phi \omega_{3}^{6}
$$

for some function $\phi$. If we put $\omega_{3}^{6}=\lambda \omega^{1}+\mu \omega^{2}$, then (8.12), (8.14) and (8.15) give

$$
\left.\begin{array}{c}
\omega_{3}^{4}=\omega_{3}^{5}=0, \quad \omega_{4}^{5}=-\frac{2 a}{\sqrt{3}} \tan \left(\frac{a u}{\sqrt{3}}\right) \omega^{2}, \\
\omega_{3}^{6}=\lambda \omega^{1}+\mu \omega^{2}, \quad \omega_{4}^{6}=\psi \lambda \omega^{1}+\psi \mu \omega^{2}, \quad \omega_{5}^{6}=\phi \lambda \omega^{1}+\phi \mu \omega^{2},
\end{array}\right\}
$$

Using (8.12), (8.13) and (8.16) we find

$$
\sqrt{3} \mu+\psi \mu=\phi \lambda, \quad \sqrt{3} \lambda-\psi \lambda=\phi \mu .
$$

Now, by differentiating the last three equations in (8.16), we obtain

$$
\begin{aligned}
e_{2} \lambda-e_{1} \mu & =-\frac{a}{\sqrt{3}} \mu \tan \left(\frac{a u}{\sqrt{3}}\right), \\
\lambda e_{2} \psi-\mu e_{1} \psi & =-\frac{2 a}{\sqrt{3}} \lambda \phi \tan \left(\frac{a u}{\sqrt{3}}\right), \\
\lambda e_{2} \phi-\mu e_{1} \phi & =\frac{2 a}{\sqrt{3}} \lambda \psi \tan \left(\frac{a u}{\sqrt{3}}\right) .
\end{aligned}
$$

Since $\lambda^{2}+\mu^{2} \neq 0,(8.17)$ gives

$$
\psi=\frac{\sqrt{3}\left(\lambda^{2}-\mu^{2}\right)}{\lambda^{2}+\mu^{2}}, \quad \phi=\frac{2 \sqrt{3} \lambda \mu}{\lambda^{2}+\mu^{2}} .
$$

Substituting (8.21) into (8.19) and (8.20) yields

$$
\begin{array}{r}
\lambda \mu\left\{\mu\left(\mu e_{1} \lambda-\lambda e_{2} \lambda\right)-\lambda\left(\mu e_{1} \mu-\lambda e_{2} \mu\right)\right\}=\frac{a \lambda^{2} \mu}{\sqrt{3}}\left(\lambda^{2}+\mu^{2}\right) \tan \left(\frac{a u}{\sqrt{3}}\right), \\
\left(\lambda^{2}-\mu^{2}\right)\left\{\mu\left(\mu e_{1} \lambda-\lambda e_{2} \lambda\right)-\lambda\left(\mu e_{1} \mu-\lambda e_{2} \mu\right)\right\}=\frac{a \lambda}{\sqrt{3}}\left(\lambda^{4}-\mu^{4}\right) \tan \left(\frac{a u}{\sqrt{3}}\right) .
\end{array}
$$

Since $\lambda^{2}+\mu^{2} \neq 0,(8.18),(8.22)$ and (8.23) imply that

$$
\begin{gathered}
\lambda_{v}=\left(\mu \cos \left(\frac{a u}{\sqrt{3}}\right)\right)_{u}, \\
3 \lambda^{2} \mu_{v}+\left(3 \cos \left(\frac{a u}{\sqrt{3}}\right) \lambda_{u}-2 \sqrt{3} a \sin \left(\frac{a u}{\sqrt{3}}\right) \lambda\right) \mu^{2}-6 \lambda \lambda_{v} \mu=\sqrt{3} a \sin \left(\frac{a u}{\sqrt{3}}\right) \lambda^{3} .
\end{gathered}
$$


Combining (8.9), (8.16), (8.21), (8.24) and (8.25), we see that the connection forms of $z: M \rightarrow \mathbb{E}^{6}$ are given by

$$
\left(\begin{array}{cccccc}
0 & q \omega^{2} & a \omega^{1} & \frac{a}{\sqrt{3}} \omega^{1} & \frac{a}{\sqrt{3}} \omega^{2} & 0 \\
-q \omega^{2} & 0 & a \omega^{2} & \frac{-a}{\sqrt{3}} \omega^{2} & \frac{a}{\sqrt{3}} \omega^{1} & 0 \\
-a \omega^{1} & -a \omega^{2} & 0 & 0 & 0 & \lambda \omega^{1}+\mu \omega^{2} \\
\frac{-a}{\sqrt{3}} \omega^{1} & \frac{a}{\sqrt{2}} \omega^{1} & 0 & 0 & 2 q \omega^{2} & \psi \lambda \omega^{1}+\psi \mu \omega^{2} \\
\frac{-a}{\sqrt{3}} \omega^{2} & \frac{-a}{\sqrt{2}} \omega^{1} & 0 & -2 q \omega^{2} & 0 & \phi \lambda \omega^{1}+\phi \mu \omega^{2} \\
0 & 0 & -\lambda \omega^{1}-\mu \omega^{2} & -\psi \lambda \omega^{1}-\psi \mu \omega^{2} & -\phi \lambda \omega^{1}-\phi \mu \omega^{2} & 0
\end{array}\right)
$$

where $q=-(a / \sqrt{3}) \tan (a u / \sqrt{3}), \lambda, \mu$ are non-trivial solutions of (8.24), (8.25), and $\phi, \psi$ are given by $(8.21)$.

By comparing connection forms (8.26) of $z$ with that of $\psi_{\lambda, \mu}^{a}$ obtained from (8.4)(8.9), we conclude that $\psi$ is congruent to $\psi_{\lambda, \mu}^{a}$ by applying the uniqueness theorem of submanifolds.

\section{Some explicit solutions of system (8.1), (8.2)}

The partial differential system (8.1), (8.2) admits infinitely many non-trivial solutions. Here we provide some explicit solutions of this system.

Example 9.1. Suppose that $\lambda=\lambda(u)$. Then (8.1) gives

$$
\mu=\sec \left(\frac{a u}{\sqrt{3}}\right) k(v)
$$

for some function $k=k(v)$. Substituting this into (8.2) gives

$$
\sqrt{3} \lambda^{2}(u) k^{\prime}(v)+\left\{\sqrt{3} \lambda^{\prime}(u)-2 a \tan \left(\frac{a u}{\sqrt{3}}\right) \lambda(u)\right\} k^{2}(v)=\frac{1}{2} a \sin \left(\frac{2 a u}{\sqrt{3}}\right) \lambda^{3}(u) .
$$

Differentiating (9.2) with respect to $v$ gives

$$
\sqrt{3} \lambda^{2}(u) k^{\prime \prime}(v)+2\left\{\sqrt{3} \lambda^{\prime}(u)-2 a \tan \left(\frac{a u}{\sqrt{3}}\right) \lambda(u)\right\} k(v) k^{\prime}(v)=0,
$$

which implies

$$
\frac{k^{\prime \prime}(v)}{2 k(u) k^{\prime}(u)}=\frac{2 a \tan (a u / \sqrt{3}) \lambda(u)-\sqrt{3} \lambda^{\prime}(u)}{\sqrt{3} \lambda^{2}(u)}=c
$$


for some constant $c$. Thus we have

$$
\begin{gathered}
k^{\prime \prime}(v)=2 c k(v) k^{\prime}(v), \\
2 a \tan \left(\frac{a u}{\sqrt{3}}\right) \lambda(u)-\sqrt{3} \lambda^{\prime}(u)=\sqrt{3} c \lambda^{2}(u) .
\end{gathered}
$$

If $c=0,(9.5)$ and (9.6) imply

$$
\lambda(u)=c_{1} \sec ^{2}\left(\frac{a u}{\sqrt{3}}\right), \quad k(v)=c_{2} v+c_{3},
$$

for some constants $c_{1}, c_{2}, c_{3}$.

If $c \neq 0$, we get from (9.6) and (9.7) that

$$
\lambda(u)=\frac{a \sec (a u / \sqrt{3})}{a c_{1} \cos (a u / \sqrt{3})+\sqrt{3} c \sin (a u / \sqrt{3})},
$$

and

$$
k(v)=\frac{1}{c_{1}-c v} \text { or } c_{2} \tan \left(c c_{2} v+c_{3}\right) \text { or }-c_{2} \tanh \left(c c_{2} v+c_{3}\right) .
$$

So, after applying a suitable translation in $v$, we have either

(i) $c=0$ and

$$
\lambda(u)=c_{1} \sec ^{2}\left(\frac{a u}{\sqrt{3}}\right), \quad k(v)=c_{2} v
$$

for some constant $c_{1}$; or

(ii) $c \neq 0$ and

$$
\begin{aligned}
\lambda(u) & =\frac{a \sec (a u / \sqrt{3})}{a c_{1} \cos (a u / \sqrt{3})+\sqrt{3} c \sin (a u / \sqrt{3})}, \\
k(v) & =\frac{1}{c v} \text { or } c_{2} \tan \left(c c_{2} v\right) \text { or }-c_{2} \tanh \left(c c_{2} v\right)
\end{aligned}
$$

for some constant $c_{2}$.

If (9.10) occurs, then (9.2) and (9.10) give $c_{1}=c_{2}=0$. Thus, we have

$$
\lambda=0, \quad \mu=b \sec \left(\frac{a u}{\sqrt{3}}\right)
$$

for some constant $b=c_{3}$.

If $(9.11)$ and $k=1 /(c v)$ hold, then we obtain from (9.2) that $c=0$, which is a contradiction. If (9.11) and $k=-c_{2} \tanh \left(c c_{2} v\right)$ hold, then (9.2) implies that $a=0$, which is also impossible. 
If (9.11) and $k=c_{2} \tan \left(c c_{2} v\right)$ hold, then (9.2) implies $c_{1}=0$ and $c_{2}=a / \sqrt{3} c$. So, we obtain

$$
\lambda=\frac{2 a}{\sqrt{3} c} \csc \left(\frac{2 a u}{\sqrt{3}}\right), \quad \mu=\frac{a}{\sqrt{3} c} \sec \left(\frac{a u}{\sqrt{3}}\right) \tan \left(\frac{a v}{\sqrt{3}}\right)
$$

Consequently, if $\lambda=\lambda(u)$, then, up to a suitable translation in $v$, the solutions of system (8.1), (8.2) are given by

$$
\begin{array}{lll}
\lambda=0, & \mu=b \sec \left(\frac{a u}{\sqrt{3}}\right), \\
\lambda=\frac{2 a}{\sqrt{3} c} \csc \left(\frac{2 a u}{\sqrt{3}}\right), & \mu=\frac{a}{\sqrt{3} c} \sec \left(\frac{a u}{\sqrt{3}}\right) \tan \left(\frac{a v}{\sqrt{3}}\right),
\end{array}
$$

for some non-zero constants $b$ and $c$.

\section{Explicit examples of non-spherical pseudo-umbilical surfaces}

Consider the solution of system (8.1), (8.2) given by

$$
\lambda=0, \quad \mu=b \sec \left(\frac{a u}{\sqrt{3}}\right)
$$

From (8.6) we have

$$
\left.\begin{array}{l}
\omega_{1}^{2}=-\frac{a}{\sqrt{3}} \tan \left(\frac{a u}{\sqrt{3}}\right) \omega^{2}, \\
\omega_{3}^{4}=\omega_{3}^{5}=\omega_{5}^{6}=0, \\
\omega_{3}^{6}=b \sec \left(\frac{a u}{\sqrt{3}}\right) \omega^{2}, \\
\omega_{4}^{5}=-\frac{2 a}{\sqrt{3}} \tan \left(\frac{a u}{\sqrt{3}}\right) \omega^{2}, \\
\omega_{4}^{6}=-\sqrt{3} b \sec \left(\frac{a u}{\sqrt{3}}\right) \omega^{2} .
\end{array}\right\}
$$

Thus, (8.9) and (10.2) imply that the immersion $\psi$ of $S^{2}(\sqrt{3} / a)$ into $\mathbb{E}^{6}$ satisfies

$$
\left.\begin{array}{l}
\psi_{u u}=a e_{3}+\frac{a}{\sqrt{3}} e_{4}, \\
\psi_{u v}=-\frac{a}{\sqrt{3}} \tan \left(\frac{a u}{\sqrt{3}}\right) x_{v}+\frac{a}{\sqrt{3}} \cos \left(\frac{a u}{\sqrt{3}}\right) e_{5}, \\
\psi_{v v}=\frac{a}{2 \sqrt{3}} \sin \left(\frac{2 a u}{\sqrt{3}}\right) x_{u}+a \cos ^{2}\left(\frac{a u}{\sqrt{3}}\right)\left(e_{3}-\frac{1}{\sqrt{3}} e_{4}\right),
\end{array}\right\}
$$




$$
\begin{aligned}
\frac{\partial e_{3}}{\partial u} & =-a x_{u}, \\
\frac{\partial e_{3}}{\partial v} & =-a x_{v}+b e_{6}, \\
\frac{\partial e_{4}}{\partial u} & =-\frac{a}{\sqrt{3}} x_{u}, \\
\frac{\partial e_{4}}{\partial v} & =\frac{a}{\sqrt{3}} x_{v}-\frac{2 a}{\sqrt{3}} \sin \left(\frac{a u}{\sqrt{3}}\right) e_{5}-\sqrt{3} b e_{6}, \\
\frac{\partial e_{5}}{\partial u} & =-\frac{a}{\sqrt{3}} \sec \left(\frac{a u}{\sqrt{3}}\right) x_{v}, \\
\frac{\partial e_{5}}{\partial v} & =-\frac{a}{\sqrt{3}} \cos \left(\frac{a u}{\sqrt{3}}\right) x_{u}+\frac{2 a}{\sqrt{3}} \sin \left(\frac{a u}{\sqrt{3}}\right) e_{4}, \\
\frac{\partial e_{6}}{\partial u} & =0, \\
\frac{\partial e_{6}}{\partial v} & =-b e_{3}+\sqrt{3} b e_{4} .
\end{aligned}
$$

By taking the derivative of the first equation in (10.3) with respect to $u$ and $v$, respectively, and by applying (10.4), we obtain

$$
\begin{aligned}
\psi_{u u u}+\frac{4}{3} a^{2} \psi_{u} & =0 \\
\psi_{u u v}+\frac{2}{3} a^{2} \psi_{v} & =-\frac{2}{3} a^{2} \sin \left(\frac{a u}{\sqrt{3}}\right) e_{5} \\
\psi_{u v v}+\frac{a^{2}}{3} \psi_{u} & =-\frac{a^{2}}{6} \sin \left(\frac{2 a u}{\sqrt{3}}\right)\left(\sqrt{3} e_{3}-3 e_{4}\right) \\
\psi_{v v v}+\frac{a^{2}}{6}\left(5+3 \cos \left(\frac{2 a u}{\sqrt{3}}\right)\right) \psi_{v} & =a^{2} \sin \left(\frac{a u}{\sqrt{3}}\right) \cos ^{2}\left(\frac{a u}{\sqrt{3}}\right) e_{5}+2 a b \cos ^{2}\left(\frac{a u}{\sqrt{3}}\right) e_{6} .
\end{aligned}
$$

Also, by differentiating (10.6) with respect to $u$, we have

$$
9 \psi_{u u u v}-6 \sqrt{3} a \psi_{u u v}-4 \sqrt{3} a^{3}\left(\tan \left(\frac{a u}{\sqrt{3}}\right)+\cot \left(\frac{a u}{\sqrt{3}}\right)\right) \psi_{v}=0 .
$$

On the other hand, solving (10.5) gives

$$
\psi=A_{1}(v) \sin \left(\frac{2 a u}{\sqrt{3}}\right)+A_{2}(v) \cos \left(\frac{2 a u}{\sqrt{3}}\right)+A_{3}(v)
$$

for some functions $A_{1}, A_{2}, A_{3}$. Substituting (10.10) into (10.9) gives $A_{2}^{\prime}(v)=A_{3}^{\prime}(v)$. Thus, we obtain $A_{3}(v)=A_{2}(v)+c$ for some constant vector $c$. We may assume that $c=0$ by applying a suitable translation if necessary. Hence, (10.10) yields

$$
\psi=A_{1}(v) \sin \left(\frac{2 a u}{\sqrt{3}}\right)+2 \cos ^{2}\left(\frac{a u}{\sqrt{3}}\right) A_{2}(v) .
$$


From (10.3) we find

$$
e_{3}=\frac{1}{6 a}\left\{3 \psi_{u u}+3 \sec ^{2}\left(\frac{a u}{\sqrt{3}}\right) \psi_{v v}-\sqrt{3} a \tan \left(\frac{a u}{\sqrt{3}}\right) \psi_{u}\right\} .
$$

Solving (10.3) and (10.8) for $e_{4}, e_{5}, e_{6}$ and using (10.4) yields

$$
\begin{aligned}
& e_{4}=\frac{1}{2 a}\left\{\sqrt{3} \psi_{u u}-\sqrt{3} \sec ^{2}\left(\frac{a u}{\sqrt{3}}\right) \psi_{v v}+a \tan \left(\frac{a u}{\sqrt{3}}\right) \psi_{u}\right\} \\
& e_{5}=\frac{1}{a} \sec \left(\frac{a u}{\sqrt{3}}\right)\left(\sqrt{3} \psi_{u v}+a \tan \left(\frac{a u}{\sqrt{3}}\right) \psi_{v}\right) \\
& e_{6}=\frac{1}{24 a b} \sec ^{2}\left(\frac{a u}{\sqrt{3}}\right)\left\{4 a^{2}\left(4+3 \cos \left(\frac{2 a u}{\sqrt{3}}\right)\right) \psi_{v}+18 \cos ^{2}\left(\frac{a u}{\sqrt{3}}\right) \psi_{u u v}+12 \psi_{v v v}\right\} .
\end{aligned}
$$

Also, differentiating (10.6) with respect to $v$ and applying (10.4) and (10.11) gives $3 A_{1}^{\prime \prime}(v)+a^{2} A_{1}(v)=0$. Hence, we have $A_{1}(v)=c_{1} \cos (a v / \sqrt{3})+c_{2} \sin (a v / \sqrt{3})$ for some constant vectors $c_{1}, c_{2}$. Therefore, (10.11) yields

$$
\psi(u, v)=c_{1} \sin \left(\frac{2 a u}{\sqrt{3}}\right) \cos \left(\frac{a v}{\sqrt{3}}\right)+c_{2} \sin \left(\frac{2 a u}{\sqrt{3}}\right) \sin \left(\frac{a v}{\sqrt{3}}\right)+2 \cos ^{2}\left(\frac{a u}{\sqrt{3}}\right) A_{2}(v) .
$$

Differentiating (10.8) with respect to $v$ and applying (10.4) and (10.16) gives

$$
3 A_{2}^{\mathrm{iv}}(v)+4\left(a^{2}+6 c^{2}\right) A_{2}^{\prime \prime}(v)+8 a^{2} c^{2} A_{2}(v)=0 .
$$

Thus, we have

$$
A_{2}=\frac{1}{2}\left(c_{3} \cos (\sqrt{\beta+\delta} v)+c_{4} \sin (\sqrt{\beta+\delta} v)+c_{5} \cos (\sqrt{\beta-\delta} v)+c_{6} \sin (\sqrt{\beta-\delta} v)\right)
$$

for some constant vectors $c_{3}, c_{4}, c_{5}, c_{6}$, where

$$
\beta=\frac{2}{3}\left(a^{2}+6 c^{2}\right), \quad \delta=\frac{2}{3} \sqrt{a^{4}+6 a^{2} c^{2}+36 c^{4}} .
$$

Therefore, (10.16) becomes

$$
\begin{aligned}
\psi(u, v)=c_{1} \sin \left(\frac{2 a u}{\sqrt{3}}\right) \cos \left(\frac{a v}{\sqrt{3}}\right)+ & c_{2} \sin \left(\frac{2 a u}{\sqrt{3}}\right) \sin \left(\frac{a v}{\sqrt{3}}\right) \\
+\cos ^{2}\left(\frac{a u}{\sqrt{3}}\right)( & c_{3} \cos (\sqrt{\beta+\delta} v)+c_{4} \sin (\sqrt{\beta+\delta} v) \\
& \left.+c_{5} \cos (\sqrt{\beta-\delta} v)+c_{6} \sin (\sqrt{\beta-\delta} v)\right) .
\end{aligned}
$$


So, after choosing some suitable initial conditions, we obtain from (10.19) that

$$
\begin{gathered}
\psi=\cos ^{2}\left(\frac{a u}{\sqrt{3}}\right)\left(\frac{\sqrt{3}}{a} \tan \left(\frac{a u}{\sqrt{3}}\right) \cos \left(\frac{a v}{\sqrt{3}}\right), \frac{\sqrt{3}}{a} \tan \left(\frac{a u}{\sqrt{3}}\right) \sin \left(\frac{a v}{\sqrt{3}}\right)\right. \\
\frac{\left(3 \delta+3 \beta-4 a^{2}\right) \sin (\sqrt{\beta-\delta} v)}{6 \delta \sqrt{\beta-\delta}}+\frac{\left(3 \delta-3 \beta+4 a^{2}\right) \sin (\sqrt{\beta+\delta} v)}{6 \delta \sqrt{\beta+\delta}} \\
\frac{\sqrt{2} a c \sin (\sqrt{\beta-\delta} v)}{\delta \sqrt{\beta+\delta}}-\frac{\sqrt{2} a c \sin (\sqrt{\beta+\delta} v)}{\delta \sqrt{\beta-\delta}}, \\
\frac{\left(2 a^{2}-3 \beta-3 \delta\right) \cos (\sqrt{\beta-\delta} v)}{4 a \delta}-\frac{\left(2 a^{2}-3 \beta+3 \delta\right) \cos (\sqrt{\beta+\delta} v)}{4 a \delta} \\
\left.\frac{\left(2 a^{2}+3 \beta+3 \delta\right) \cos (\sqrt{\beta-\delta} v)}{4 \sqrt{3} a \delta}-\frac{\left(2 a^{2}+3 \beta-3 \delta\right) \cos (\sqrt{\beta+\delta} v)}{4 \sqrt{3} a \delta}\right) .
\end{gathered}
$$

For any $a, c>0$, it is easy to verify that $\psi$ is a constant isotropic pseudo-umbilical immersion of an open subset of $S^{2}(\sqrt{3} / a)$ into $\mathbb{E}^{6}$. Such immersions have constant mean curvature $a$ and non-parallel mean curvature vector. Moreover, because $\langle\psi, \psi\rangle=3 \cos ^{2}(a u / \sqrt{3}) / a^{2}$, such immersions are non-spherical.

Remark 10.1. The above examples illustrate that the class of surfaces of Case (2)(b) in Theorem 7.1 is large.

Remark 10.2. The surfaces given by (10.20) are the first explicit examples of pseudoumbilical surfaces in Euclidean spaces which are neither minimal nor minimal in any hypersphere.

Remark 10.3. For further examples of surfaces in Euclidean spaces with contact number $\geqslant 5$ and their characterization, see [8].

Acknowledgements. S.-J.L. is supported by the NSFC (project 19771039) and the PSSF (project 960179) of Guangdong Province, China. S.-J.L. expresses his hearty thanks to Professor Bang-Yen Chen for his kind help and encouragement and to colleagues at MSU for the hospitality he received during his visit in the summer of 2002.

\section{References}

1. K. Arslan And A. West, Product submanifolds with pointwise 3-planar normal sections, Glasgow Math. J. 37 (1995), 73-81.

2. K. Arslan And A. West, Non-spherical submanifolds with pointwise 2-planar normal sections, Bull. Lond. Math. Soc. 28 (1996), 88-92.

3. A. L. BESSE, Manifolds all of whose geodesics are closed (Springer, 1979).

4. R. L. Bryant, Minimal surfaces of constant curvature in $S^{n}$, Trans. Am. Math. Soc. 290 (1985), 259-271.

5. B.-Y. Chen, Minimal surfaces with constant Gauss curvature, Proc. Am. Math. Soc. 34 (1972), 504-508.

6. B.-Y. Chen, Geometry of submanifolds (Dekker, New York, 1973).

7. B.-Y. Chen, Differential geometry of submanifolds with planar normal sections, Annli Mat. Pura Appl. 130 (1982), 59-66. 
8. B.-Y. ChEn, Flat surfaces with high contact number and their characterization, submitted.

9. B.-Y. Chen And J.-M. Morvan, Géométrie des surfaces Lagrangiennes de $\mathbb{C}^{2}$, J. Math. Pures Appl. 66 (1987), 321-335.

10. B.-Y. Chen AND P. VERHEyen, Submanifolds with geodesic normal sections, Math. Ann. 269 (1984), 417-429.

11. W. Dal Lago, A. N. García And C. U. SÁnchez, Planar normal sections on the natural imbedding of a flag manifold, Geom. Dedicata 53 (1994), 223-235.

12. W. Dal Lago, A. N. García And C. U. SÁnchez, Projective subspaces in the variety of normal sections and tangent spaces to a symmetric space, J. Lie Theory 8 (1998), $415-428$.

13. W. Dal Lago, A. N. García And C. U. SÁnchez, Maximal projective subspaces in the variety of planar normal sections of a flag manifold, Geom. Dedicata 75 (1999), 219-233.

14. J. Deprez AND P. VERheYen, Immersions with circular normal sections and normal sections of product immersions, Geom. Dedicata 20 (1986), 335-344.

15. J. Deprez, P. Verheyen And L. Verstraelen, Higher-dimensional normal sections, An. Sttiint. Univ. Al. I. Cuza Iaşi Ia 31 (1985), 255-259.

16. D. Hoffmann And R. Osserman, The geometry of the generalized Gauss map, Memoirs of the American Mathematical Society, vol. 236 (1980).

17. Y. Hong, On submanifolds with planar normal sections, Michigan Math. J. 32 (1985), 203-210.

18. Y. Hong, Helical submanifolds in Euclidean spaces, Indiana Univ. Math. J. 35 (1986), $29-43$.

19. C. S. Houn AND G. Q. WANG, Isotropic submanifolds with pointwise planar normal sections, J. Geom. 26 (1986), 99-104.

20. Y. H. KIM, Minimal surfaces of pseudo-Euclidean spaces with geodesic normal sections, Diff. Geom. Applic. 5 (1995), 321-329.

21. S.-J. LI, Isotropic submanifolds with pointwise 3-planar normal sections, Boll. UMI B 7 (1987), 373-385.

22. B. O’NeILl, Isotropic and Kaehler submanifolds, Can. J. Math. 17 (1965), 907-915.

23. M. Pinl, Minimalflächen fester Gaußscher Krümmung, Math. Ann. 136 (1958), 34-40.

24. K. Sakamoto, Helical immersions into a unit sphere, Math. Ann. 261 (1982), 63-80.

25. C. U. SÁnchez, A. N. García And W. DAL LAgo, Planar normal sections on the natural embedding of a real flag manifold, Beiträge Algebra Geom. 41 (2000), 513-530.

26. P. Verheyen, Submanifolds with geodesic normal sections are helical, Rend. Sem. Mat. Univ. Polit. Torino 43 (1985), 511-527. 